\title{
The Gaia-ESO Survey: Galactic evolution of lithium from iDR6 ${ }^{\star \star \star \star}$
}

\author{
D. Romano ${ }^{1}$, L. Magrini ${ }^{2}$, S. Randich ${ }^{2}$, G. Casali ${ }^{2,3}$, P. Bonifacio ${ }^{4}$, R. D. Jeffries ${ }^{5}$, F. Matteucci ${ }^{6,7,8}$, E. Franciosini ${ }^{2}$, \\ L. Spina ${ }^{9}$, G. Guiglion ${ }^{10}$, C. Chiappini ${ }^{10}$, A. Mucciarellii ${ }^{11,1}$, P. Ventura ${ }^{12}$, V. Grisoni ${ }^{13,7}$, M. Bellazzini ${ }^{1}$, T. Bensby ${ }^{14}$, \\ A. Bragaglia' ${ }^{1}$, P. de Laverny ${ }^{15}$, A. J. Korn ${ }^{16}$, S. L. Martell ${ }^{17,18}$, G. Tautvaišiené ${ }^{19}$, G. Carraro ${ }^{20}$, A. Gonneau ${ }^{21}$, \\ P. Jofré22 , E. Pancino ${ }^{2,23}$, R. Smiljanic ${ }^{24}$, A. Vallenari ${ }^{9}$, X. Fu ${ }^{25}$, M. L. Gutiérrez Albarrán ${ }^{26}$, F. M. Jiménez-Esteban ${ }^{27}$, \\ D. Montes ${ }^{26}$, F. Damiani ${ }^{28}$, M. Bergemann ${ }^{29}$, and C. Worley ${ }^{21}$
}

(Affiliations can be found after the references)

Received 19 May 2021 / Accepted 18 June 2021

\begin{abstract}
Context. After more than 50 years, astronomical research still struggles to reconstruct the history of lithium enrichment in the Galaxy and to establish the relative importance of the various ${ }^{7} \mathrm{Li}$ sources in enriching the interstellar medium (ISM) with this fragile element.

Aims. To better trace the evolution of lithium in the Milky Way discs, we exploit the unique characteristics of a sample of open clusters (OCs) and field stars for which high-precision ${ }^{7} \mathrm{Li}$ abundances and stellar parameters are homogeneously derived by the Gaia-ESO Survey (GES).

Methods. We derive possibly un-depleted ${ }^{7} \mathrm{Li}$ abundances for $26 \mathrm{OCs}$ and star forming regions with ages from young ( $\left.\sim 3 \mathrm{Myr}\right)$ to old ( 4.5 Gyr), spanning a large range of galactocentric distances, $5<R_{\mathrm{GC}} / \mathrm{kpc}<15$, which allows us to reconstruct the local late Galactic evolution of lithium as well as its current abundance gradient along the disc. Field stars are added to look further back in time and to constrain ${ }^{7} \mathrm{Li}$ evolution in other Galactic components. The data are then compared to theoretical tracks from chemical evolution models that implement different ${ }^{7} \mathrm{Li}$ forges.

Results. Thanks to the homogeneity of the GES analysis, we can combine the maximum average ${ }^{7} \mathrm{Li}$ abundances derived for the clusters with ${ }^{7} \mathrm{Li}$ measurements in field stars. We find that the upper envelope of the ${ }^{7} \mathrm{Li}$ abundances measured in field stars of nearly solar metallicities $(-0.3<[\mathrm{Fe} / \mathrm{H}] / \mathrm{dex}<+0.3)$ traces very well the level of lithium enrichment attained by the ISM as inferred from observations of cluster stars in the same metallicity range. We confirm previous findings that the abundance of ${ }^{7} \mathrm{Li}$ in the solar neighbourhood does not decrease at supersolar metallicity. The comparison of the data with the chemical evolution model predictions favours a scenario in which the majority of the ${ }^{7} \mathrm{Li}$ abundance in meteorites comes from novae. Current data also seem to suggest that the nova rate flattens out at later times. This requirement might have implications for the masses of the white dwarf nova progenitors and deserves further investigation. Neutrino-induced reactions taking place in core-collapse supernovae also produce some fresh lithium. This likely makes a negligible contribution to the meteoritic abundance, but could be responsible for a mild increase in the ${ }^{7} \mathrm{Li}$ abundance in the ISM of low-metallicity systems that would counterbalance the astration processes.
\end{abstract}

Key words. Galaxy: abundances - Galaxy: evolution - Galaxy: stellar content - stars: abundances - open clusters and associations: general nuclear reactions, nucleosynthesis, abundances

\section{Introduction}

Of all the elements in the periodic table, the main isotope of lithium, ${ }^{7} \mathrm{Li}$, has without any doubt the most complex origin and evolution. Its nuclei were produced in significant amounts during Big Bang nucleosynthesis (BBN), 10 per cent directly as ${ }^{7} \mathrm{Li}$ in the first few minutes and 90 per cent as ${ }^{7} \mathrm{Be}$ that decayed to ${ }^{7} \mathrm{Li}$ later on (Steigman 2007; Khatri \& Sunyaev 2011; Fields et al. 2020). Thereafter, both spallation processes that take place in the interstellar medium (ISM) and nuclear burning in stars contribute to increase its abundance from the primordial value to that currently observed in meteorites and young T Tauri stars.

It is a little disconcerting that none of the proposed ${ }^{7} \mathrm{Li}$ production channels have been firmly assessed yet. The factor of three difference between the primordial abundance of lithium predicted by the standard BBN (SBBN) theory assuming the Planck baryon density, $A(\mathrm{Li})_{\mathrm{P}}^{\text {th }} \sim 2.7$ dex (Pitrou et al. 2018), and that inferred from observations of halo stars on the 'Spite

\footnotetext{
* Based on data products from observations made with ESO Telescopes at the La Silla Paranal Observatory under programmes 188.B3002, 193.B-0936, and 197.B-1074.

$\star \star$ Full Tables 1 and 3 are only available at the CDS via anonymous ftp to cdsarc.u-strasbg. $f r(130.79 .128 .5)$ or via http://cdsarc. u-strasbg.fr/viz-bin/cat/J/A+A/xxx/Ayyy
}

plateau' that should not have depleted their ${ }^{7} \mathrm{Li}, A(\mathrm{Li})_{\mathrm{P}}^{\mathrm{obs}} \sim 2.2$ dex (Spite \& Spite 1982; Bonifacio \& Molaro 1997; Sbordone et al. 2010), constitutes the well-known cosmological lithium problem and raises the question as to whether the BBN model (e.g. Coc et al. 2012; Goudelis et al. 2016; Hou et al. 2017; Luo et al. 2019; Clara \& Martins 2020) or stellar physics (Michaud et al. 1984; Charbonnel \& Primas 2005; Mucciarelli et al. 2012; Nordlander et al. 2012; Fu et al. 2015; Gao et al. 2020, and references therein) is to blame.

As ${ }^{7} \mathrm{Li}$ enters the chemical composition of successive stellar generations, it is swiftly burned through ${ }^{7} \mathrm{Li}(\mathrm{p}, \alpha){ }^{4} \mathrm{He}$ reactions already on the pre-main sequence wherever the temperature exceeds about $2.5 \times 10^{6} \mathrm{~K}$. For ${ }^{7} \mathrm{Li}$ to be produced in stars, it is necessary that ${ }^{7} \mathrm{Be}$ be formed first, at temperatures exceeding $4 \times 10^{7} \mathrm{~K}$. The newly formed ${ }^{7} \mathrm{Be}$ must then be promptly carried by convection to cooler layers, where it decays to ${ }^{7} \mathrm{Li}$ that is preserved and, eventually, ejected. This mechanism was first proposed by Cameron (1955) and Cameron \& Fowler (1971) to explain the existence of Li-rich asymptotic giant branch (AGB) stars. Low-mass stars on the first ascent of the red giant branch (RGB) may also show enhanced lithium if some extra deep mixing is associated with the cool bottom processing (Sackmann \& Boothroyd 1999). Although some Li-rich and very Li-rich giant stars do exist (Wallerstein \& Sneden 1982; Ruchti et al. 2011; 
Silva Aguirre et al. 2014; Kirby et al. 2016; Smiljanic et al. 2018; Singh et al. 2019; Charbonnel et al. 2020; Deepak et al. 2020), low- and intermediate-mass stars are unlikely to contribute significantly to the ${ }^{7} \mathrm{Li}$ enrichment on galactic scales (Ventura et al. 2000, 2020) unless some extreme assumptions are made on the effectiveness of mass loss along the giant branches that should be maximal exactly when the stars are Li-rich (Romano et al. 2001; Travaglio et al. 2001).

Things change if low- and intermediate-mass stars are hosted in cataclysmic variables. Classical nova explosions occur in these binary systems when the white dwarf (WD) primary accretes matter from a secondary star that overflows its Roche lobe. Once the temperature of the deepest layers accreted on top of the WD exceeds $\sim 7 \times 10^{7} \mathrm{~K}$, a thermonuclear runaway is initiated, which leads to an outburst that does not disrupt the WD. After the ejection of the envelope, which is enriched in rare isotopes, the process is re-initiated (José \& Hernanz 2007, and references therein); on average, the typical nova experiences $10^{4}$ outbursts during its lifetime (Bath \& Shaviv 1978).

Although theoretical calculations of ${ }^{7} \mathrm{Li}$ production during thermonuclear runaways demonstrated long ago that novae could be important contributors to the Galactic ${ }^{7} \mathrm{Li}$ enrichment (Starrfield et al. 1978; D'Antona \& Matteucci 1991), until very recently there was no observational evidence to support the theory. Izzo et al. (2015) and Tajitsu et al. (2015) first detected, respectively, the blueshifted $\mathrm{Li}$ I $\lambda 6708 \AA$ line in the early spectra of nova V1369 Cen and strong Be II features in the spectra of nova V339 Del. As ${ }^{7}$ Be was detected in more objects (e.g. Selvelli et al. 2018; Molaro et al. 2020b), a discrepancy between the observational abundance estimates and theoretical predictions of nucleosynthesis models became apparent, with the former exceeding the latter by up to one order of magnitude. The reason for this may reside in the (wrong) assumption of equality between the relative fractions of the ions $\mathrm{Be}$ II/Be and $\mathrm{Ca}$ II/Ca in the nova ejecta (Chugai \& Kudryashov 2020) and/or in an overly simplistic treatment of the complex structure of the ejecta itself (Shore \& De Gennaro Aquino 2020). However, Denissenkov et al. (2021) show that an enhanced abundance of ${ }^{4} \mathrm{He}$ in nova envelopes would push the ${ }^{7} \mathrm{Be}$ abundance predicted by nova models closer to the observed values. From the above it seems evident that at present neither spectroscopic observations nor stellar models can provide us with a sound, quantitative estimate of ${ }^{7} \mathrm{Li}$ production from nova systems.

The flux of neutrinos that emerges from the collapsing core of an exploding massive star is another trigger of ${ }^{7} \mathrm{Li}$ synthesis (see Woosley et al. 1990, for the first realistic exploration of the $v$-process in stars). Sieverding et al. (2018) have presented results for a grid of solar-metallicity models with stellar masses in the range $13-30 \mathrm{M}_{\odot}$. Their models take into account up-to-date cross-sections for the neutrino-induced reactions and a more realistic treatment of the neutrino transport in supernova (SN) simulations that results in lower-energy spectra for all neutrino families. Due to the reduction in neutrino energies, the efficiency of the $v$-process is diminished. Their ${ }^{7} \mathrm{Li}$ yields range from $1 \times 10^{-8} \mathrm{M}_{\odot}$ to $2 \times 10^{-8} \mathrm{M}_{\odot}$, one order of magnitude lower than the older estimates by Woosley \& Weaver (1995). However, the use of time-dependent neutrino emission spectra and neutrino oscillation and metallicity effects are expected to affect ${ }^{7} \mathrm{Li}$ production in the $v$-process, and larger yields could be obtained (Kusakabe et al. 2019; Sieverding et al. 2019). Spectroscopic observations of $\mathrm{Li}$ in $\mathrm{SN}$ remnants could establish the mechanism of ${ }^{7} \mathrm{Li}$ production in massive stars. The only measurement of this kind we are aware of (Taylor et al. 2012) finds no evidence of ${ }^{7} \mathrm{Li}$ synthesis by neutrino-induced spallation in core-collapse SNe. However, the absence of evidence is not evidence of absence; clearly, we need to probe many more diffuse molecular clouds near other SN remnants.

We conclude this roundup of ${ }^{7} \mathrm{Li}$ factories by briefly reviewing another triplet of possible sources. First, high-energy Galactic cosmic ray (GCR) nuclei impacting stationary ISM atoms have been known for 50 years as ${ }^{7} \mathrm{Li}$ sources (Reeves et al. 1970; Meneguzzi et al. 1971). However, they produce no more than 20-25 per cent of the meteoritic ${ }^{7} \mathrm{Li}$ abundance (see Romano et al. 2001; Prantzos 2012). Second, spallation in hot advectiondominated accretion flows can make quiescent soft X-ray transients quite efficient at producing ${ }^{7} \mathrm{Li}$ (Yi \& Narayan 1997; Fujimoto et al. 2008, and references therein). Yet, under reasonable hypotheses, the contribution from these objects to ${ }^{7} \mathrm{Li}$ pollution on galactic scales turns out to be negligible (Romano et al. 2001). A similar conclusion is reached regarding the creation of lithium in flares of active stars (Kelly et al. 2020, and references therein).

The Galactic chemical evolution (GCE) models adopted in this work take all of the ${ }^{7} \mathrm{Li}$ sources mentioned above, bar the last two, into account. The models predict the amount of lithium a star will be formed with at any given time for different Galactic components. The stellar abundance then falls over time, which makes the upper envelope of the lithium abundances measured in warm, dwarf stars the useful thing to measure (see below). The trends of $A(\mathrm{Li})$ as functions of $[\mathrm{Fe} / \mathrm{H}]$ and age predicted for solar neighbourhood stars are compared to spectroscopic data from the sixth internal data release (iDR6) of the Gaia-ESO Spectroscopic Survey ${ }^{1}$ (GES; Gilmore et al. 2012; Randich et al. 2013). Moreover, we compare, for the first time, the theoretical $A(\mathrm{Li})$ gradient to ${ }^{7} \mathrm{Li}$ abundances measured in objects covering a large range of galactocentric distances. After Rebolo et al. (1988), it became common practice to assume that the upper envelope of the abundances of ${ }^{7} \mathrm{Li}$ measured in warm, dwarf Galactic stars tracks the ${ }^{7} \mathrm{Li}$ enrichment of the ISM fairly faithfully. In more recent years, the mean values of the highest ${ }^{7} \mathrm{Li}$ abundances in bins of metallicity have been put forward as a more appropriate indicator (e.g. Lambert \& Reddy 2004; Delgado Mena et al. 2015; Guiglion et al. 2016; Fu et al. 2018). It has also become clear that the mixing of different populations can lead to spurious trends; in particular, the effects of stellar radial migration must be taken into account (Guiglion et al. 2019). Finally, it has been shown that the trends of ${ }^{7} \mathrm{Li}$ with metallicity, age, and galactocentric distance are best traced when considering cluster stars that have not undergone any depletion (see Randich et al. 2020).

This paper is organised as follows. Section 2 details the sample selection, ${ }^{7} \mathrm{Li}$ abundance determination, and the kinematical and dynamical analysis. We introduce the adopted GCE model in Sect. 3. Section 4 describes the evolution of ${ }^{7} \mathrm{Li}$ in the thick and thin discs emerging from the field and cluster star samples selected from GES iDR6. Lithium observations are compared to the predictions of chemical evolution models in Sect. 4 and further discussed in Sect. 5. Finally, in Sect. 6 we draw our conclusions.

\section{Dataset}

This study is based on the last internal data release of the GES. We take advantage of the ${ }^{7} \mathrm{Li}$ abundances and stellar parameters homogeneously determined for pre-main-sequence, mainsequence, turn-off, and sub-giant stars observed with the multiobject optical fibre facility FLAMES (Fibre Large Array Multi Element Spectrograph; Pasquini et al. 2002) in the Milky Way

1 https://WWW.gaia-eso . eu 
Romano et al.: Galactic evolution of lithium from GES iDR6

Table 1. Stellar parameters and lithium abundances of 3210 stars in the field, ordered by increasing age.

\begin{tabular}{|c|c|c|c|c|c|c|c|c|c|c|c|c|c|c|c|}
\hline GES name & $\begin{array}{c}\text { RA } \\
\text { [deg] }\end{array}$ & $\begin{array}{c}\text { Dec } \\
\text { [deg] }\end{array}$ & $\begin{array}{l}T_{\text {eff }} \\
{[\mathrm{K}]}\end{array}$ & $\begin{array}{c}\delta T_{\text {eff }} \\
{[\mathrm{K}]}\end{array}$ & $\begin{array}{l}\log g \\
{[\operatorname{dex}]}\end{array}$ & $\begin{array}{c}\log g \\
{[\operatorname{dex}]}\end{array}$ & $\begin{array}{c}\mathrm{Fe} / \mathrm{H}] \\
{[\mathrm{dex}]}\end{array}$ & $\begin{array}{c}\delta[\mathrm{Fe} / \mathrm{H}] \\
{[\mathrm{dex}]}\end{array}$ & $\begin{array}{l}A(\mathrm{Li}) \\
{[\mathrm{dex}]}\end{array}$ & $\begin{array}{c}\delta(\mathrm{Li}) \\
{[\mathrm{dex}]}\end{array}$ & $\begin{array}{c}\alpha / \mathrm{Fe}] \\
{[\mathrm{dex}]}\end{array}$ & $\begin{array}{c}\delta[\alpha / \mathrm{Fe}] \\
{[\mathrm{dex}]}\end{array}$ & $\begin{array}{c}\text { Age } \\
{[\mathrm{Gyr}]}\end{array}$ & $\begin{array}{l}\delta \text { Age } \\
{[\mathrm{Gyr}]}\end{array}$ & $\begin{array}{l}R_{\mathrm{GC}} \\
{[\mathrm{pc}]}\end{array}$ \\
\hline $17530917-2937418$ & 268.28821 & -29.6283 & 6807 & 66 & 4.30 & 0.17 & 0.18 & 0.06 & $<2.62$ & - & - & - & 1.06 & 0.56 & 6009 \\
\hline $17524903-2926366$ & 268.20429 & -29.4435 & 6754 & 67 & 4.45 & 0.18 & 0.08 & 0.05 & $<2.28$ & - & - & - & 1.13 & 0.61 & 5916 \\
\hline $10371153-5838408$ & 159.29804 & -58.6447 & 6721 & 67 & 4.23 & 0.17 & 0.24 & 0.05 & 3.36 & 0.06 & - & - & 1.18 & 0.60 & 7683 \\
\hline $17530416-2936240$ & 268.26733 & -29.6067 & 6887 & 65 & 4.24 & 0.17 & 0.04 & 0.05 & 2.49 & 0.19 & - & - & 1.19 & 0.60 & 4419 \\
\hline $17525280-2931432$ & 268.22000 & -29.5287 & 6785 & 66 & 4.25 & 0.18 & 0.15 & 0.05 & 2.97 & 0.06 & - & - & 1.19 & 0.61 & 6164 \\
\hline$\ldots$ & $\ldots$ & $\ldots$ & $\ldots$ & $\ldots$ & $\ldots$ & $\ldots$ & $\ldots$ & $\ldots$ & $\ldots$ & $\ldots$ & $\ldots$ & $\ldots$ & $\ldots$ & $\ldots$ & $\ldots$ \\
\hline
\end{tabular}

Notes. The reported errors are the result of the GES homogenisation procedure and include random and systematic error sources, apart from the uncertainties on ${ }^{7} \mathrm{Li}$ abundances (see text) and on age determinations (which were computed as the average of the half widths of the 68\% confidence intervals calculated by aussieq2). The table is available in its entirety at the CDS. A portion is shown here for guidance regarding its form and contents.

field and in open clusters (OCs). In particular, regarding the determination of ${ }^{7} \mathrm{Li}$, OC stars were observed either with the Ultraviolet and Visual Echelle Spectrograph $\left(\mathrm{UVES}^{2}\right)$ and the 580 setup or with GIRAFFE and the HR15N setup; both configurations include the lithium lines. The HR15N grating was not employed for observations of stars in GES field samples; however, targets observed in cluster fields that were later recognised as non-members may have their ${ }^{7} \mathrm{Li}$ abundances derived from GIRAFFE spectra. Our GES iDR6 star sample was additionally cross-matched with the Gaia Early Data Release 3 (EDR3) catalogue (Gaia Collaboration et al. 2016, 2020) to perform a full chemo-dynamical characterisation of the stars.

\subsection{Lithium abundance determination and sample selection}

One-dimensional (1D) local thermodynamical equilibrium (LTE) abundances of ${ }^{7} \mathrm{Li}$ in GES iDR6 were obtained from equivalent width measurements of the $\operatorname{Li}$ I $\lambda 6708 \AA$ spectral feature. The abundance derivation procedure (Franciosini et al., in prep.) involved the use of a new set of curves of growth specifically defined for the GES that relies on the grid of synthetic spectra described in Guiglion et al. (2016, see also de Laverny et al. 2012); for GIRAFFE spectra, where the lithium feature is blended with the nearby Fe I $\lambda 6707.4 \AA$ line, a consistent correction was applied. Upper limits were derived when the line was not detectable; more specifically, the larger of the measured equivalent width and the associated uncertainty was assigned as upper limit when the line was not detected or barely visible.

We did not correct the ${ }^{7} \mathrm{Li}$ abundances for 3D non-LTE effects, since they are almost negligible for the majority of the stars in our sample, barely reaching -0.1 dex for a handful of stars (see Magrini et al. 2021, their Figs. 1 and 2). According to recent work by Wang et al. (2021), a systematic -0.1 dex correction applies to metal-rich dwarfs with $T_{\text {eff }}$ between 5000 and $6500 \mathrm{~K}$. The correction then decreases to zero between 6500 and $7000 \mathrm{~K}$. For the coolest, metal-rich pre-main-sequence stars in clusters the corrections may be as severe as -0.3 dex, but these values are very uncertain, since they rely on extrapolations of extant grids.

The field stars used in the present paper were selected as: (i) field stars that are non-members of the old and intermediateage OCs (age $>120 \mathrm{Myr}$ ), taking into account all stars not selected as member stars on the basis of their radial velocities from the GES, proper motions, and parallaxes from Gaia EDR3, and (ii) stars observed in the GES field samples, as indicated by the keywords $G E S \_M W, G E S \_M W \_B L, G E S \_K 2, G E S \_C R$ in the

\footnotetext{
2 See Smiljanic et al. (2014) and Lanzafame et al. (2015) for a complete description of the analysis of the FLAMES-UVES spectra of, respectively, FGK and pre-main-sequence stars in the GES.
}

field $G E S \_F L D$. We combined the two samples, applying a further selection on stellar parameter uncertainties $\left(\delta T_{\text {eff }}<100 \mathrm{~K}\right.$, $\delta \log g<0.2 \mathrm{dex}, \delta[\mathrm{Fe} / \mathrm{H}]<0.15 \mathrm{dex})$ and including both stars with measured lithium abundances with error on $A(\mathrm{Li})$ lower than 0.25 dex and upper limits. We cross-matched our catalogue with Gaia EDR3 and computed the stellar luminosities using the geometric distances from Bailer-Jones et al. (2021) and the $G$ magnitudes of Gaia, converted into $V$ magnitudes using the colours $G_{\mathrm{BP}}$ and $G_{\mathrm{RP}}$, as stated in Magrini et al. (2021). To compute the bolometric magnitudes we used the bolometric corrections $\mathrm{BC}(K)$ tabulated by Alonso et al. (1999, their Table 5), which are based on $V-K$ colours, and $K$ magnitudes from 2MASS (Skrutskie et al. 2006).

After removing the giant stars from the sample, we were left with 6207 late-type stars with effective temperatures $\left(T_{\text {eff }}\right)$ in the range $5300-7000 \mathrm{~K}$, surface gravities $(\log g$ ) of 3.5 to 4.6 (in cgs units), and metallicities $-1.5<[\mathrm{Fe} / \mathrm{H}] / \mathrm{dex}<+0.5$, which were observed with either the FLAMES-UVES 580 setup at high resolution $(R \simeq 47000)$ or the FLAMES-GIRAFFE HR15N setup at medium resolution $(R \simeq 19000)$. To further improve the quality of the sample, we retained only the stars with the highestquality spectra (i.e. signal-to-noise ratio $S / N \geq 50$ ). This almost halved our working sample, which finally consists of 3210 stars. Stars with $\log g \simeq 3.5$ dex may undergo the first dredge up and, thus, display lithium abundances lower than the initial value. Yet, our sample contains only 7 stars with $3.5<\log g<3.7$, which makes the exact $\log g$ cut rather irrelevant.

We estimated the age for each star of our working sample using the aussieq 2 tool $^{3}$ that is an extension of the qoyllurquipu $\left(q^{2}\right)$ Python package (Ramírez et al. 2014). It calculates stellar ages (and masses) by isochrone fitting, starting from the stellar parameters $\left(T_{\text {eff }}, \log g\right.$, and $V$ magnitude) and a grid of isochrones, also taking into account the uncertainties on the stellar parameters. The difference between the observed parameters and the corresponding values in the model grid is used as a weight to calculate the probability distribution function; the most probable age, that is, the peak of the probability distribution, is obtained through a maximum likelihood calculation (see also Casali et al. 2020). For this work, we adopted the YalePotsdam Stellar Isochrones (YaPSI; Spada et al. 2017) and took the $\alpha$-enhancement effects on the model atmospheres into account, following the procedure outlined in Casali et al. (2020, their Sect. 2.3).

The recommended GES iDR6 stellar parameters (from spectroscopy), lithium abundances and $[\alpha / \mathrm{Fe}]^{4}$ ratios, along with their errors, for the selected stars are reported in Cols. 4 to 13 of Table 1. In general, within the GES collaboration the stellar

\footnotetext{
3 https://github.com/spinastro/aussieq2

${ }^{4}[\alpha / \mathrm{Fe}]=([\mathrm{Ti} / \mathrm{Fe}]+[\mathrm{Ca} / \mathrm{Fe}]+[\mathrm{Si} / \mathrm{Fe}]+[\mathrm{Mg} / \mathrm{Fe}]) / 4$.
} 
A\&A proofs: manuscript no. 41340corr

Table 2. Parameters and average maximum lithium abundance for the selected clusters, sorted by increasing age.

\begin{tabular}{|c|c|c|c|c|c|c|}
\hline Cluster & $\begin{array}{c}\text { Age } \\
\text { [Gyr] }\end{array}$ & $\begin{array}{r}R_{\mathrm{GC}} \\
{[\mathrm{kpc}]}\end{array}$ & $\begin{array}{c}{[\mathrm{Fe} / \mathrm{H}]} \\
{[\mathrm{dex}]}\end{array}$ & $\begin{array}{c}A(\mathrm{Li})_{\max } \\
{[\mathrm{dex}]}\end{array}$ & \# of stars & $\begin{array}{c}T_{\text {eff }} \text { range } \\
{[\mathrm{K}]}\end{array}$ \\
\hline$\rho \mathrm{Oph}^{a}$ & 0.003 & 7.88 & $(-0.265)$ & $3.28 \pm 0.13$ & 25 & $3006-4536$ (pre-main sequence) \\
\hline Alessi 43 & 0.011 & 8.18 & $+0.02 \pm 0.06$ & $3.27 \pm 0.22$ & 46 & 3513-5135 (pre-main sequence) \\
\hline $25 \mathrm{Ori}^{b}$ & 0.013 & 8.31 & $(0.02)$ & $3.18 \pm 0.14$ & 7 & 3119-3386 (pre-main sequence) \\
\hline Collinder 197 & 0.014 & 8.20 & $(0.02)$ & $3.21 \pm 0.15$ & 64 & 3515-4441 (pre-main sequence) \\
\hline NGC 2232 & 0.018 & 8.27 & $(0.005)$ & $3.22 \pm 0.11$ & 14 & 5031-6891 (young) \\
\hline NGC 2547 & 0.032 & 8.05 & $(-0.055)$ & $3.35 \pm 0.10$ & 8 & 5748-6286 (young) \\
\hline IC 4665 & 0.033 & 7.71 & $(0.00)$ & $3.44 \pm 0.10$ & 5 & 5674-6133 (young) \\
\hline NGC 6405 & 0.035 & 7.54 & $(-0.01)$ & $3.31 \pm 0.08$ & 7 & $5848-6419$ \\
\hline IC 2602 & 0.036 & 7.95 & $(-0.01)$ & $3.31 \pm 0.13$ & 4 & 5766-6438 (young) \\
\hline Blanco 1 & 0.105 & 7.96 & $-0.12 \pm 0.07$ & $3.15 \pm 0.04$ & 7 & 5923-6476 (young) \\
\hline NGC 6067 & 0.126 & 6.16 & $+0.03 \pm 0.05$ & $3.38 \pm 0.09$ & 15 & $6518-8000$ \\
\hline NGC 6709 & 0.190 & 7.22 & $-0.03 \pm 0.06$ & $3.31 \pm 0.01$ & 2 & $6628-7410$ \\
\hline NGC 2516 & 0.240 & 7.98 & $-0.04 \pm 0.05$ & $3.33 \pm 0.07$ & 3 & $6531-6839$ \\
\hline Berkeley 30 & 0.295 & 13.59 & $-0.15 \pm 0.05$ & $3.11 \pm 0.17$ & 14 & $6630-6980$ \\
\hline NGC 6705 & 0.309 & 6.02 & $+0.02 \pm 0.01$ & $3.34 \pm 0.02$ & 7 & $6673-6984$ \\
\hline NGC 3532 & 0.398 & 7.85 & $-0.01 \pm 0.05$ & $3.25 \pm 0.07$ & 4 & $6825-6865$ \\
\hline NGC 6802 & 0.660 & 6.71 & $+0.14 \pm 0.04$ & $3.32 \pm 0.02$ & 2 & 6677-6919 \\
\hline NGC 2355 & 1.000 & 9.84 & $-0.07 \pm 0.05$ & $3.17 \pm 0.09$ & 6 & $6716-6975$ \\
\hline Berkeley 81 & 1.148 & 5.21 & $+0.22 \pm 0.05$ & $3.39 \pm 0.11$ & 1 & 6836 \\
\hline Berkeley 73 & 1.413 & 14.97 & $-0.26 \pm 0.05$ & $3.16 \pm 0.11$ & 8 & $6721-6885$ \\
\hline Berkeley 44 & 1.445 & 6.58 & $+0.22 \pm 0.05$ & $3.21 \pm 0.10$ & 9 & $6564-6761$ \\
\hline NGC 2158 & 1.549 & 13.18 & $-0.16 \pm 0.05$ & $3.19 \pm 0.09$ & 5 & $6585-6889$ \\
\hline Ruprecht 134 & 1.660 & 5.44 & $+0.27 \pm 0.05$ & $3.47 \pm 0.04$ & 8 & $6697-6879$ \\
\hline NGC 2420 & 1.738 & 10.49 & $-0.16 \pm 0.05$ & $3.19 \pm 0.07$ & 22 & 6378-6769 (turn-off) \\
\hline Trumpler 20 & 1.862 & 6.82 & $+0.13 \pm 0.04$ & $3.29 \pm 0.12$ & 7 & $6700-6928$ \\
\hline NGC 2243 & 4.365 & 11.00 & $-0.44 \pm 0.05$ & $2.94 \pm 0.10$ & 7 & 6064-6314 (post turn-off) \\
\hline
\end{tabular}

Notes. Ages were taken from Cantat-Gaudin et al. (2020). Galactocentric distances were computed with the astropy package (see Sect. 2.2). Average $[\mathrm{Fe} / \mathrm{H}]$ from UVES members in GES iDR6 are reported for clusters older than $100 \mathrm{Myr}$, while for younger objects median (and more uncertain) $[\mathrm{Fe} / \mathrm{H}]$ are given in brackets. The error bars associated with the average maximum ${ }^{7} \mathrm{Li}$ abundances represent the standard deviation of the stars used to compute the mean. ${ }^{(a)}$ Star forming region. Age and galactocentric distance from Spina et al. (2017). ${ }^{(b)}$ ASCC 16 in Cantat-Gaudin et al. (2020).

spectra were processed by different analysis nodes, each adopting the same model atmospheres and line lists though (Heiter et al. 2015), which provided different measurements affected by different biases and random errors (Smiljanic et al. 2014). In order to homogenise the results, Gaia benchmark stars were used as reference objects to identify the bias function of each node. In GES iDR6, the errors provided by the various nodes were not used - the errors associated with the recommended values were mainly estimated as internal errors of the method. Lithium abundances, however, were determined only by the Arcetri analysis node (Franciosini et al., in prep.). For this element, the associated uncertainties were derived by combining in quadrature the uncertainties due to the errors on each stellar parameter and on the measured equivalent width (the latter obtained using the formula by Cayrel 1988). The ages and the galactocentric distances (computed as specified in Sect. 2.2) of objects in our wellcontrolled sample of Milky Way field stars are listed in the last three columns of Table 1 .

The field star sample was complemented with estimates of the average maximum ${ }^{7} \mathrm{Li}$ abundance ${ }^{5}$ in selected OCs. Ages and distances of OC members are, in general, much more accurate than for isolated field stars. Moreover, young OCs did not have time to travel significantly away from their birthplaces. Therefore, their chemical composition is representative of the history of chemical enrichment of their environs. The GES iDR6 has delivered parameters and abundances for members of 87 OCs and star forming regions, including 20 objects for which spectra

\footnotetext{
5 This is the average of the highest ${ }^{7} \mathrm{Li}$ abundances that are measured in possibly un-depleted cluster members.
}

were retrieved from the ESO archive and 2 clusters used for calibration only (i.e. not overlapping with the science sample; see Pancino et al. 2017). For young clusters present in the previous internal data release of the survey (iDR5), GES spectroscopy and Gaia astrometry were combined to assign membership probabilities (see Jackson et al. 2020); targets with probability $P>0.9$ were selected as cluster members. For a few young clusters not present in GES iDR5, the membership was established based only on radial velocity. For intermediate-age and old clusters, the members were selected as in Magrini et al. (2021), deriving first the peak and standard deviation of the radial velocity distribution and selecting objects within $2 \sigma$ of the peak. Then, the average parallax and proper motion with the corresponding standard deviations were calculated for the selected objects and a $2 \sigma$ clipping was applied. By using this method, we found an excellent agreement with the results of Cantat-Gaudin et al. (2020) for the clusters in common.

Following the criteria outlined by Randich et al. (2020) and briefly recalled below, we restricted our analysis to 26 clusters including one star forming region with age less than $5 \mathrm{Myr}$ and several young OCs (age $<100 \mathrm{Myr}$ ), paying attention to select for each cluster only the members that suffered minimal lithium depletion, if any (see Table 2). In the first place, we selected very young clusters with ${ }^{7} \mathrm{Li}$ detections in at least four to five members that supposedly reflect the original ${ }^{7} \mathrm{Li}$ abundance of their parent clouds. Clusters younger than 100 Myr host premain-sequence or zero-age main-sequence stars that should not have depleted any ${ }^{7} \mathrm{Li}$. As a matter of fact, though, ${ }^{7} \mathrm{Li}$ depletion may show up in cool members of clusters as young as $5 \mathrm{Myr}$ 
Table 3. Orbital parameters for the selected clusters and field stars, sorted by increasing age.

\begin{tabular}{lccccccc}
\hline \hline Cluster name/GES name & $\begin{array}{c}U \\
{\left[\mathrm{~km} \mathrm{~s}^{-1}\right]}\end{array}$ & $\begin{array}{c}V \\
{\left[\mathrm{~km} \mathrm{~s}^{-1}\right]}\end{array}$ & $\begin{array}{c}W \\
{\left[\mathrm{~km} \mathrm{~s}^{-1}\right]}\end{array}$ & $\begin{array}{c}J_{r} \\
{\left[\mathrm{kpc} \mathrm{km} \mathrm{s}^{-1}\right]}\end{array}$ & $\begin{array}{c}J_{z} \\
{\left[\mathrm{kpc} \mathrm{km} \mathrm{s}^{-1}\right]}\end{array}$ & $\begin{array}{c}L_{r} \\
{\left[\mathrm{kpc} \mathrm{km} \mathrm{s}^{-1}\right]}\end{array}$ & $\begin{array}{c}L_{z} \\
{\left[\mathrm{kpc} \mathrm{km} \mathrm{s}^{-1}\right]}\end{array}$ \\
\hline Alessi 43 & -21.69 & 212.26 & -0.43 & 7.871 & 0.095 & 10.792 & 1724.73 \\
25 Ori & -6.55 & 224.21 & 2.94 & 1.488 & 0.316 & 18.832 & 1863.66 \\
Collinder 197 & -22.63 & 214.22 & -1.92 & 7.770 & 0.086 & 8.823 & -1744.27 \\
NGC 2232 & -14.01 & 215.96 & -4.49 & 3.047 & 0.152 & 3.651 & 1785.27 \\
NGC 2547 & -5.20 & 221.92 & -3.94 & 0.501 & 0.147 & 7.550 & 1783.70 \\
$\ldots$ & $\ldots$ & $\ldots$ & $\ldots$ & $\ldots$ & $\ldots$ & $\ldots$ & $\ldots$ \\
\hline \multicolumn{7}{c}{. Field stars } \\
\hline 17530917-2937418 & -21.46 & 223.21 & -11.88 & 4.453 & 0.785 & 10.015 & 1341.21 \\
$17524903-2926366$ & -29.35 & 237.86 & -2.50 & 12.317 & 0.118 & 9.626 & 1407.15 \\
$10371153-5838408$ & -34.89 & 182.58 & -8.17 & 47.240 & 0.415 & 2.812 & 1340.94 \\
$17530416-2936240$ & 20.21 & 217.83 & 9.66 & 3.166 & 0.990 & 19.701 & 962.62 \\
$17525280-2931432$ & 36.47 & 238.86 & -16.62 & 19.449 & 1.417 & 8.693 & 1472.30 \\
$\ldots$ & $\ldots$ & $\ldots$ & $\ldots$ & $\ldots$ & $\ldots$ & $\ldots$ & $\ldots$ \\
\hline
\end{tabular}

Notes. Columns 2 to 4 report the galactocentric velocity components; Cols. 5 and 6 give the radial and vertical actions, respectively; Cols. 7 and 8 report the radial and vertical components of the angular momentum. The table is available in its entirety at the CDS. A portion is shown here for guidance regarding its form and contents.

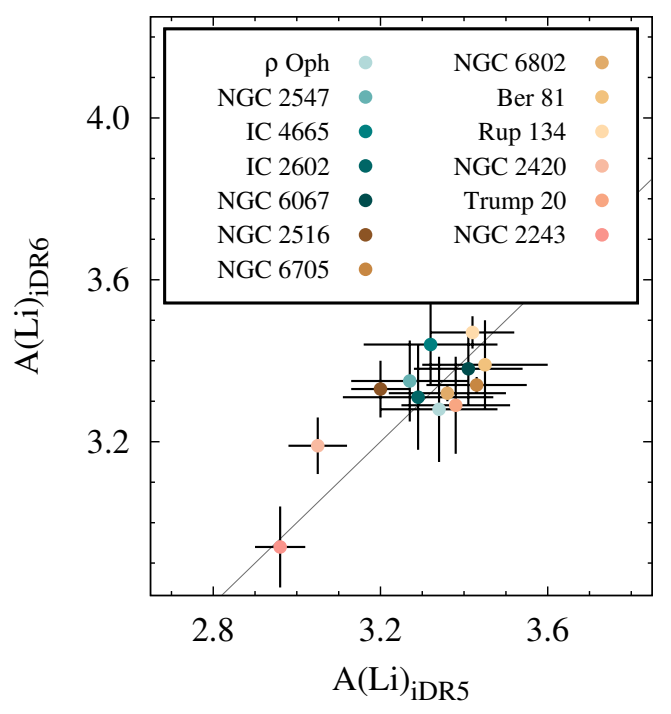

Fig. 1. Average maximum ${ }^{7} \mathrm{Li}$ abundances from iDR6 (this work) and iDR5 (Randich et al. 2020) for the clusters in common.

(see e.g. Bouvier et al. 2016; Jeffries et al. 2021, and references therein). The average maximum ${ }^{7} \mathrm{Li}$ abundance for the young clusters considered in this work was computed selecting only the stars that trace the upper envelope of the $A(\mathrm{Li})$ versus $T_{\text {eff }}$ distributions, for GES clusters sampled well enough, which maximises our chances to recover the initial, un-depleted value. We added to this sample clusters in which stars on the blue (warm) side of the 'lithium dip' (Boesgaard \& Tripicco 1986) were observed, either on the main sequence or after the turn-off, before surface dilution in connection with the first dredge-up starts. The only exception is NGC 2243 that, because of its older age ( $\simeq 4.4 \mathrm{Gyr}$, all the other clusters being younger than $2 \mathrm{Gyr}$ ), is likely to display only a lower limit to the actual original value. With this caveat in mind, we retained this cluster to increase the statistics in the outer disc. The average maximum ${ }^{7} \mathrm{Li}$ abundance, $A(\mathrm{Li})_{\max }$, along with the associated dispersion, the age, galactocentric distance and metallicity adopted for each cluster are listed in Table 2. The number of stars used to calculate $A(\mathrm{Li})_{\max }$ and their $T_{\text {eff }}$ range are also given in Cols. 6 and 7. Notwithstanding the relative paucity of objects that passed our selection criteria (30 per cent of all OCs in GES iDR6), we can still probe a wide galactocentric distance baseline, $5<R_{\mathrm{GC}} / \mathrm{kpc}<15$, which makes it possible to do a meaningful test of the predictions of the GCE model regarding the existence and extent of any ${ }^{7} \mathrm{Li}$ gradient across the Milky Way disc.

For most clusters in common, the average maximum ${ }^{7} \mathrm{Li}$ abundances displayed in Table 2 differ slightly (see Fig. 1) from the corresponding values reported in Table 1 of Randich et al. (2020). This is mainly due to the improved abundance measurements in iDR6 with respect to the previous internal data release of the GES on which the work of Randich et al. is based, though in some cases the number of member stars with ${ }^{7} \mathrm{Li}$ determinations used to compute the mean has been reduced. It is further noted that some very young clusters studied by Randich et al. (2020) - namely, NGC 6530, Trumpler 14, Chamaeleon I, and NGC 2264 - show a large scatter in their revised ${ }^{7} \mathrm{Li}$ abundances, possibly due to stellar activity or accretion, while a few interesting objects included in iDR6 (e.g. NGC 6649 and NGC 6281) are still missing a clean membership analysis. These clusters are not part of the present study.

\subsection{Kinematic and dynamical properties of OCs and field stars}

We set the distance of the Sun from the Galactic centre to $R_{0}=$ $8 \mathrm{kpc}$, its height above the plane to $z_{0}=0.025 \mathrm{kpc}$ (Jurić et al. 2008; Bovy et al. 2012), and used the astrometric data from Gaia to transform the celestial coordinates of stars and OCs in our sample in galactocentric radius and height above the Galactic plane using astropy ${ }^{6}$ (Astropy Collaboration et al. 2013). The radial velocities from GES iDR6 and parallaxes and proper motions from Gaia were used to obtain the orbital parameters and actions of clusters and field stars in our working sample with the galpy package ${ }^{7}$ (Bovy 2015). For the Milky Way's gravitational potential, we assumed the code built-in model MWpotential2014. The local standard of rest (LSR) velocity was set to $V_{\mathrm{LSR}}=220 \mathrm{~km} \mathrm{~s}^{-1}$ (Bovy et al. 2012) and we as-

\footnotetext{
6 https://WWW . astropy . org/

7 https://github.com/jobovy/galpy
} 
sumed $(U, V, W)_{\odot}=(11.1,12.24,7.25) \mathrm{km} \mathrm{s}^{-1}$ for the velocity of the Sun relative to the LSR (Schönrich et al. 2010). The orbital parameters of our sample clusters and stars that we exploit in Sect. 4.5 are listed in Table 3.

\section{Chemical evolution model}

This section provides an overview of the adopted GCE model. More information can be found in the original papers.

\subsection{General features}

In order to study the evolution of lithium in the Milky Way, we adopted the 'parallel GCE model' presented by Grisoni et al. $(2017,2018)$. In the parallel model, the thick- and thin-disc components of our Galaxy are formed on different timescales out of two distinct episodes of infall of primordial gas and evolved independently from each other (see Ferrini et al. 1992 and Chiappini 2009, for former approaches to the parallel galaxy formation scenario).

The contemporary evolution of the two components causes their stellar populations to overlap in a wide range of metallicities, while the less (more) efficient star formation assumed for the thin (thick) disc leads to distinct sequences of lower (higher) $[\alpha / \mathrm{Fe}]$ ratios in the $[\alpha / \mathrm{Fe}]$ versus $[\mathrm{Fe} / \mathrm{H}]$ plane, as observed (see Grisoni et al. 2017, and references therein). The interested reader is referred to Grisoni et al. $(2017,2018)$ for a detailed description of the main assumptions and underlying equations of the model. In the following, we focus on the adopted nucleosynthesis prescriptions, with special emphasis on those for ${ }^{7} \mathrm{Li}$.

\subsection{Nucleosynthesis prescriptions}

The nucleosynthesis prescriptions are the same as model MWG11 of Romano et al. (2019). For low- and intermediate-mass stars with initial masses in the range $1 \leq m / \mathrm{M}_{\odot}<9$, we adopted the yields by Ventura et al. $(2013,2020)$ that include a proper treatment of the super-AGB phase for the most massive AGB stars. For massive stars with $13 \leq m / \mathbf{M}_{\odot} \leq 100$, we adopted the yields by Limongi \& Chieffi (2018). In particular, for $[\mathrm{Fe} / \mathrm{H}] \leq-1$ dex we used their 'set R' (see the original paper for details) and assumed that all massive stars are fast rotators, whilst for $[\mathrm{Fe} / \mathrm{H}]>-1$ dex we used the yields from the non-rotating stellar models. With these choices we are able to fit the abundance measurements of several chemical elements in the solar neighbourhood as well as across the whole Milky Way disc (see Romano et al. 2019, 2020; Grisoni et al. 2020, Baratella et al., in prep.; Grisoni et al., in prep.). Thus, we believe that the adopted yield set provides a robust backbone for general GCE studies. Since the $v$-process is not included in the computations of Limongi \& Chieffi (2018), to take account of the effects of neutrino-induced nucleosynthesis on the evolution of ${ }^{7} \mathrm{Li}$ in the Galaxy we resorted to the ${ }^{7} \mathrm{Li}$ yields by Woosley \& Weaver $(1995)^{8}$. Following arguments by Duncan et al. (1997), we lowered the original yields by a factor of two (see discussion in Romano et al. 2001).

\footnotetext{
8 Although other authors (e.g. Yoshida et al. 2008; Sieverding et al. 2018, 2019; Kusakabe et al. 2019) provide up-to-date estimates of the $v$-process ${ }^{7} \mathrm{Li}$ yield using cutting-edge $\mathrm{SN}$ explosion and $\mathrm{SN}$ neutrino models, as well as new cross-sections for the relevant reactions, the work by Woosley \& Weaver (1995) remains the only one to offer a grid of yields that span a range of stellar masses and metallicities adequate for use in GCE studies.
}

The stellar yields for type Ia SNe (exploding WDs in binary systems) were taken from Iwamoto et al. (1999, their model W7). Type Ia SNe are responsible for the bulk of Fe production at late times and dominate the decrease in the $[\alpha / \mathrm{Fe}]$ ratio at high metallicities, but do not produce any ${ }^{7} \mathrm{Li}$. Classical novae, instead, are possibly important ${ }^{7} \mathrm{Li}$ factories. The nova outburst rate was implemented in the parallel GCE code following the scheme outlined in Romano et al. (1999, see also D'Antona \& Matteucci 1991 and Matteucci et al. 1995). The fiducial model adopted in this work assumes that nearly 2 per cent of all stars with initial mass in the range 1-8 $\mathrm{M}_{\odot}$ enter the formation of nova systems. Unless otherwise stated, the average ${ }^{7} \mathrm{Li}$ production per nova outburst is set to $M_{\mathrm{Li} \text {, burst }}^{\text {nova }}=2.55 \times 10^{-10} \mathrm{M}_{\odot}$, consistent with the direct estimate of the ${ }^{7} \mathrm{Li}$ yield from observations of nova V1369 Cen by Izzo et al. (2015) and with the results from some recent hydrodynamical simulations (Starrfield et al. 2020). When assuming that each nova system suffers $10^{4}$ outbursts during its lifetime (Bath \& Shaviv 1978), the above number translates into a total ejected mass of ${ }^{7} \mathrm{Li}$ per nova of $M_{\mathrm{Li} \text {, tot }}^{\text {nova }} \simeq 2.5 \times 10^{-6} \mathrm{M}_{\odot}$. We explore the effects of deviations from the main assumptions of this fiducial model in Sect. 4.4.

Cosmic-ray bombardment of the ISM also creates ${ }^{7} \mathrm{Li}$. The contribution of GCR spallation was taken into account by incorporating the absolute yields by Lemoine et al. (1998). We took the yields corresponding to the lower-bound spectrum in their Table 1. Alternatively, one could use empirical yields inferred from ${ }^{9} \mathrm{Be}$ observations in stars to find similar results (see Grisoni et al. 2019, and references therein).

\section{Results}

In this section we exploit the GES iDR6 dataset presented in Sect. 2 and the GCE model introduced in Sect. 3 to study the evolution of lithium in the Milky Way. We first present the trends of $A(\mathrm{Li})$ with stellar parameters for our sample of field stars, divided into inner disc, solar neighbourhood, and outer disc inhabitants according to their current positions along the disc. In Sect. 4.2, we focus on ${ }^{7} \mathrm{Li}$ enrichment in the solar vicinity. Solar neighbourhood stars in our sample are separated into four distinct populations via chemical selection criteria and their properties are compared to the predictions of the parallel GCE model. We then extend our modelling to the whole Milky Way disc to investigate the Galactic ${ }^{7} \mathrm{Li}$ gradient. Lastly, we use the Toomre diagram and a diagram of angular momentum, $L_{z}$, and square root of the radial action, $\sqrt{J_{r}}$ (see Feuillet et al. 2020), to assess the membership of the stars to the thick or thin discs on dynamical bases and to pinpoint possibly accreted stars. The lithium content of a few candidate accreted stars is discussed in comparison to the results of models that trace the evolution of ${ }^{7} \mathrm{Li}$ in local dwarf galaxies (Matteucci et al. 2021).

\subsection{Tracing the evolution of lithium in the Milky Way}

Figures 2 and 3 show, respectively, the $T_{\text {eff }}$ versus age and Kiel diagrams for our selection of field stars with ${ }^{7} \mathrm{Li}$ determinations from high-quality spectra $(\mathrm{S} / \mathrm{N} \geq 50)$ in GES iDR6 catalogue. In agreement with previous studies (see e.g. Bensby \& Lind 2018, for recent work), we find that: (i) due to the weakness of the ${ }^{7} \mathrm{Li}$ line, several high-temperature stars have only upper limits on their ${ }^{7} \mathrm{Li}$ abundance; (ii) ${ }^{7} \mathrm{Li}$ measurements in stars on the lower main sequence witness efficient lithium destruction in the deeper convective envelopes of the cooler stars; (iii) the highest ${ }^{7} \mathrm{Li}$ abundances, $A(\mathrm{Li})>3.0 \mathrm{dex}$, are found in the upper main 


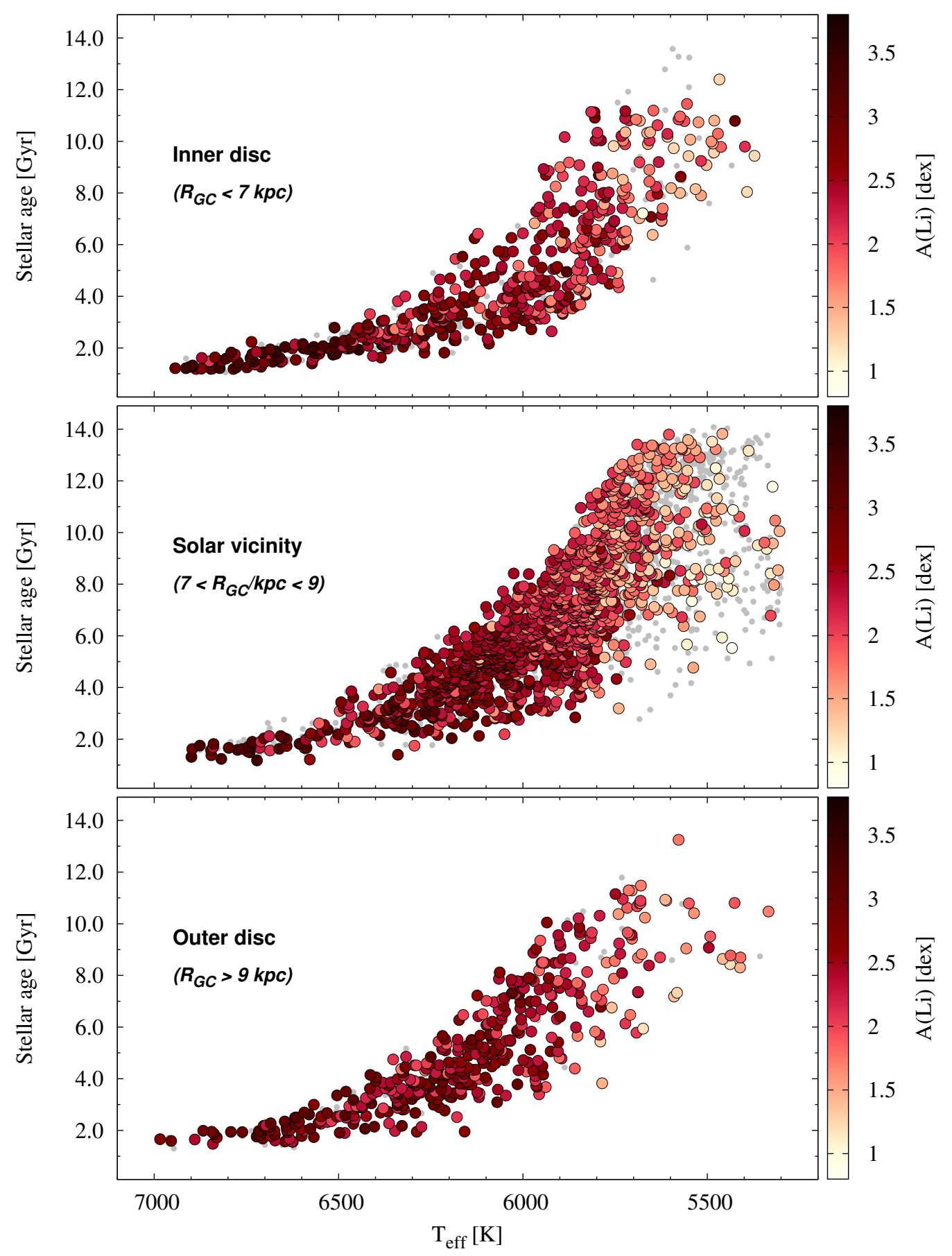

Fig. 2. Effective temperature versus stellar age diagrams for the GES iDR6 sample of field stars discussed in this paper, divided into inner disc (top panel, 619 stars), solar vicinity (middle panel, 2129 stars), and outer disc sub-samples (bottom panel, 462 stars). Stellar ages were determined from isochrone fitting using the aussieq2 code and YaPSI stellar models (Spada et al. 2017). Stars with lithium measurements are colour-coded according to their ${ }^{7} \mathrm{Li}$ abundance. Grey dots represent upper limits.

sequence and around the turn-off; (iv) in the lithium dip region, the stars span the full range of ${ }^{7} \mathrm{Li}$ abundances, from the Spite plateau value to very close to the meteoritic one, with a few objects characterised by anomalously low lithium abundances; (v) the hotter the stars, the less we can go back in time. Referring to the last point, we note that our field star sample does not contain any objects younger than $1 \mathrm{Gyr}$, due to GES selection criteria (see also Thompson et al. 2018). We further remark that the ages of the cool stars are very uncertain, because of the fact that the isochrones tend to crowd together. However, this does not impact the present study, since we are mostly interested in the stars that potentially preserve their original ${ }^{7} \mathrm{Li}$ and the cool stars are known to efficiently destroy it.

After careful scrutiny of Figs. 2 and 3, we are led to suggest that, among the field stars, only those with $T_{\text {eff }} \geq 6800$ $6900 \mathrm{~K}$ can be safely used as tracers of ${ }^{7} \mathrm{Li}$ evolution. Stars with $6500<T_{\text {eff }} / \mathrm{K}<6800$ could still be employed, but one should consider their location in a Kiel diagram very carefully. With- 


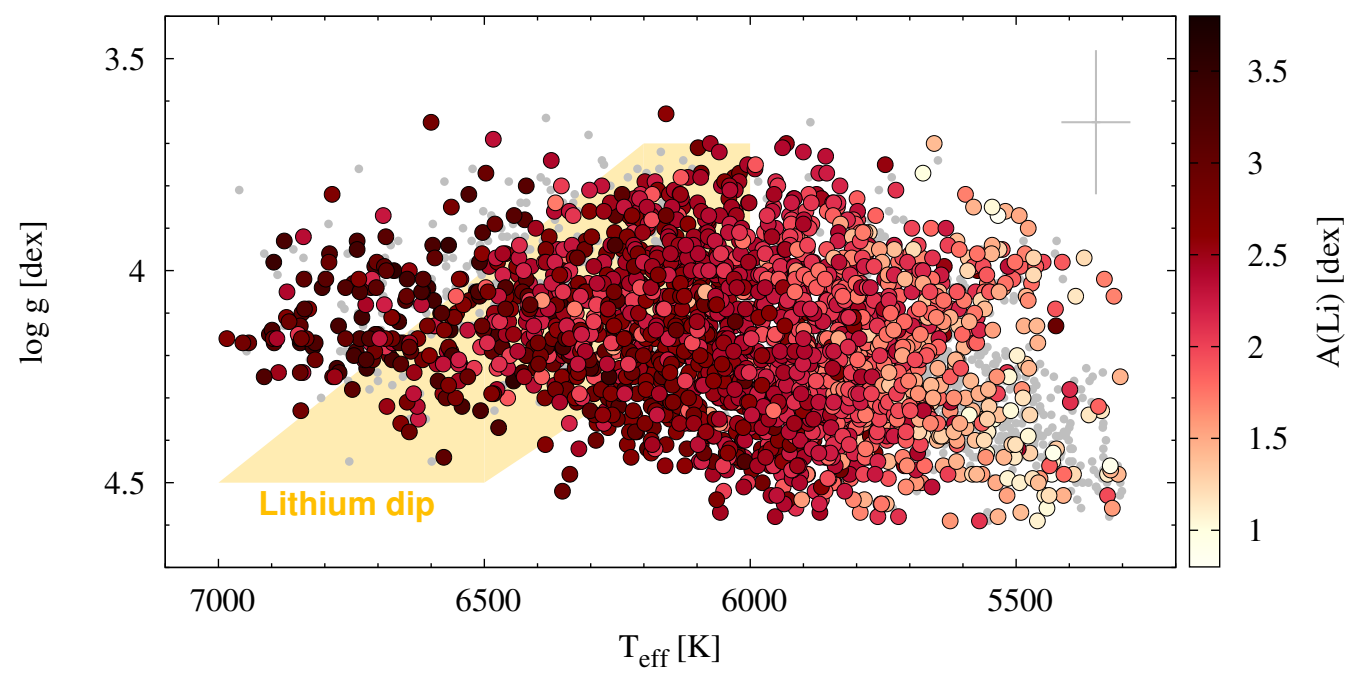

Fig. 3. Effective temperature versus gravity diagram for the full GES iDR6 sample of field stars discussed in this paper. Stars with lithium measurements are colour-coded according to their ${ }^{7} \mathrm{Li}$ abundance. Grey dots represent upper limits. The approximate location of the ${ }^{7} \mathrm{Li}$ dip region (see also Gao et al. 2020) is highlighted (yellow area).

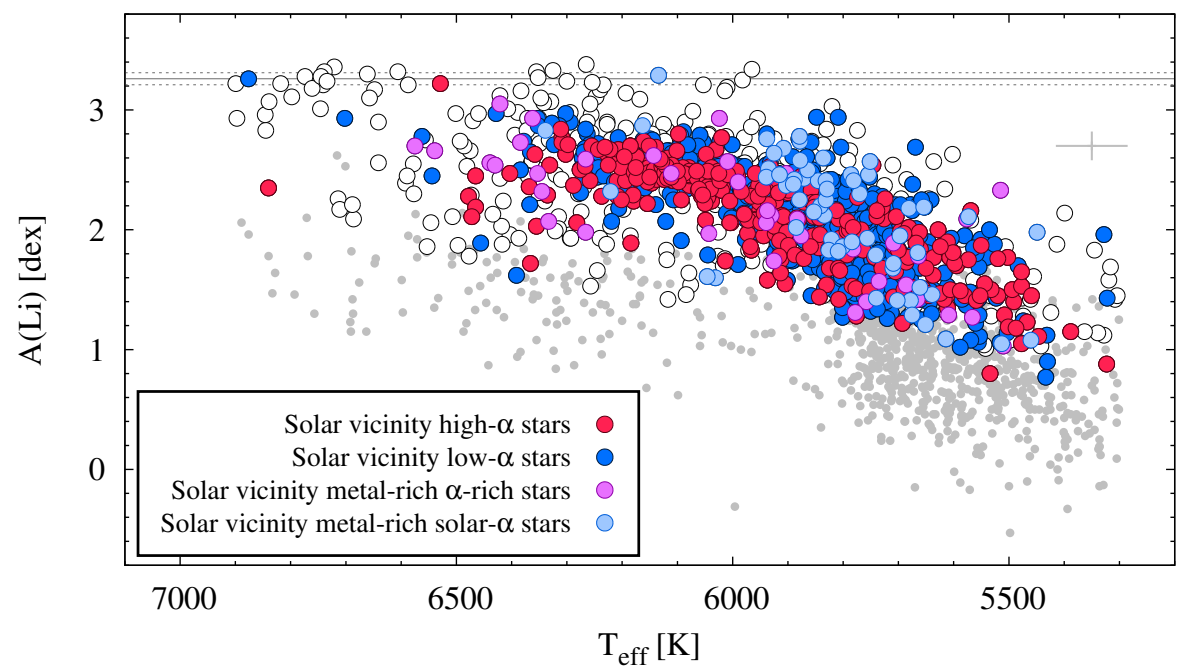

Fig. 4. Lithium abundance as a function of effective temperature for our sample of GES iDR6 field stars restricted to targets found in the solar neighbourhood $\left(7<R_{\mathrm{GC}} / \mathrm{kpc}<9\right)$. Both ${ }^{7} \mathrm{Li}$ measurements (circles) and upper limits (grey dots) are reported. Whenever possible, high- $\alpha$ (red circles), low- $\alpha$ (blue), metal-rich $\alpha$-rich (magenta), and super metal-rich solar- $\alpha$ stars (light blue) were identified according to their position in the $[\alpha / \mathrm{Fe}]-[\mathrm{Fe} / \mathrm{H}]$ diagram, adopting the same criteria as Guiglion et al. $(2016,2019$, see also Recio-Blanco et al. 2014). Empty circles represent stars without chemical identification. The grey lines show the ${ }^{7} \mathrm{Li}$ content in meteorites (3.26 \pm 0.05 , Lodders et al. 2009).

out a severe temperature cut, the commonly adopted approach of binning the data in metallicity to compute mean values of $A(\mathrm{Li})$ for the stars with the highest ${ }^{7} \mathrm{Li}$ abundances in each metallicity bin will lead to a spurious $A(\mathrm{Li})$ versus $[\mathrm{Fe} / \mathrm{H}]$ trend either way, with the computed values unavoidably reflecting stellar depletion to some extent (Anthony-Twarog et al. 2018). Our suggestion is consistent with the findings of Gao et al. (2020). These authors separate nearly 63000 late-type stars with ${ }^{7} \mathrm{Li}$ abundances obtained in the framework of the Galactic Archeology with HERMES (GALAH) survey into two groups - one on the warm side, the other on the cool side of the lithium dip - and analyse the behaviour of each group in the $A(\mathrm{Li})-[\mathrm{Fe} / \mathrm{H}]$ plane. They suggest that the warm stars trace effectively the evolution of ${ }^{7} \mathrm{Li}$ in the Galaxy, while the cool stars have burnt lithium to a large degree (see also Charbonnel et al. 2021).

In Fig. 4 we display the run of $A(\mathrm{Li})$ with $T_{\text {eff }}$ for stars currently found in the solar neighbourhood. Our sample contains 1293 solar neighbourhood stars with ${ }^{7} \mathrm{Li}$ measurements (for 836 targets we could only give upper limits) that belong to chemically distinct populations. We adopted the criteria presented in Recio-Blanco et al. (2014) and applied later to lithium studies in Guiglion et al. $(2016,2019)$ and identified 311 high- $\alpha$ stars, 524 low- $\alpha$ stars, a metal-rich $\alpha$-rich population (also called mr $\alpha$, Adibekyan et al. 2011) consisting of 41 stars, and a super metalrich solar- $\alpha$ population $(61 \text { objects })^{9}$. We did not classify 356

\footnotetext{
${ }^{9}$ For the sake of brevity, we name stars displaying high $[\alpha / \mathrm{Fe}]$ ratios, high- $\alpha$ stars, those displaying low $[\alpha / \mathrm{Fe}]$ ratios, low- $\alpha$ stars, etcetera.
} 


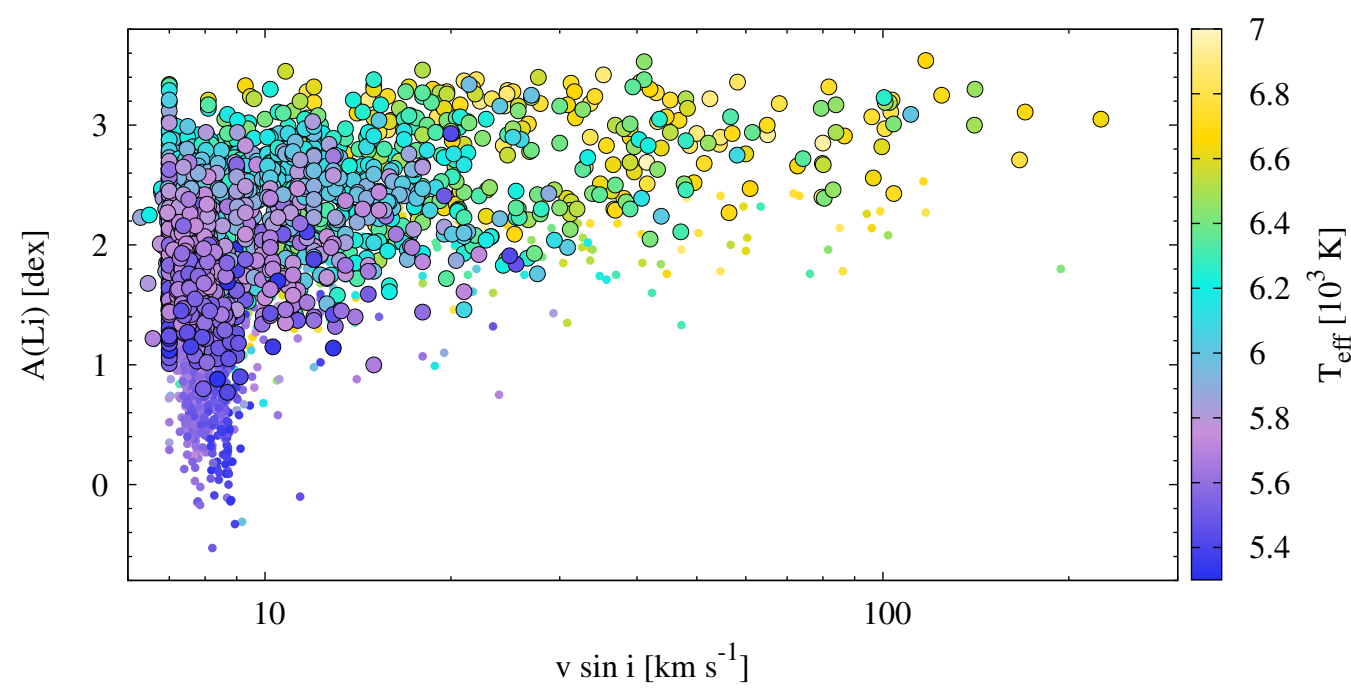

Fig. 5. Lithium abundance as a function of rotational velocity for the full field GES iDR6 stellar sample selected for this paper (3210 objects). The stars are colour-coded according to their effective temperature. Both ${ }^{7} \mathrm{Li}$ measurements (circles) and upper limits (dots) are shown. Rotational velocities are measured from FLAMES-UVES spectra; typical errors on $v \sin i$ are $\leq 5 \mathrm{~km} \mathrm{~s}^{-1}$.

stars that lack simultaneous measurements of the elements used to define the $[\alpha / \mathrm{Fe}]$ ratios in this work $(\mathrm{Mg}, \mathrm{Si}, \mathrm{Ca}, \mathrm{Ti}$; empty circles in Fig. 4). All the sub-populations are characterised by similar $A(\mathrm{Li})$ spreads at a given temperature (when considering only the temperature range in which all populations are well sampled). In the range $5500<T_{\text {eff }} / \mathrm{K}<6300$, the upper envelope of the ${ }^{7} \mathrm{Li}$ abundances traced by the high- $\alpha$ stars lies systematically below that traced by the other components. If the high- $\alpha$ stars were all born in the thick-disc component, this could indicate that the thick disc attained a lower level of ${ }^{7} \mathrm{Li}$ enrichment (see Guiglion et al. 2016; Grisoni et al. 2019). This conclusion is reinforced by an inspection of the $A(\mathrm{Li})$ versus $[\mathrm{Fe} / \mathrm{H}]$ diagram (see next section). We further note that there is a temperature threshold, $T_{\text {eff }} \simeq 6000 \mathrm{~K}$, above which the stars with the highest ${ }^{7} \mathrm{Li}$ abundances in our solar vicinity sample share the same (meteoritic) abundance; below such a threshold the abundance of lithium decreases steadily with decreasing temperature, as expected.

We also looked for possible correlations between ${ }^{7} \mathrm{Li}$ abundance and rotation in our sample of field stars. Figure 5 shows the $A(\mathrm{Li})-v \sin \mathrm{i}$ plane, where $v \sin \mathrm{i}$ is the projected equatorial rotational velocity, for all the 3210 stars with high-quality ${ }^{7} \mathrm{Li}$ abundances in our sample colour-coded in accordance with their effective temperature. Unsurprisingly, the hottest (more massive) stars in the sample have the highest rotational velocities. For $v \sin \mathrm{i}>30 \mathrm{~km} \mathrm{~s}^{-1}$, there is a spread of about $1 \mathrm{dex}$ in ${ }^{7} \mathrm{Li}$ abundances. The stars in this region of the diagram are all F-type stars; their atmospheres are expected to be mostly in radiative equilibrium and to show minimal convection effects. We interpret the observed spread as due to rotational mixing; the few F-type stars with low $v$ sin i could have a low inclination angle. For $v \sin \mathrm{i} \leq 20 \mathrm{~km} \mathrm{~s}^{-1}$ a much larger spread in ${ }^{7} \mathrm{Li}$ abundance is seen, driven by convection. The few stars with $A(\mathrm{Li})$ abundances in excess of 3.4 dex are all relatively young (ages $~ 1-2$ Gyr) and located in the inner disc, where the GCE model predicts a current ISM ${ }^{7} \mathrm{Li}$ abundance higher than in the solar neighbourhood. By sure, such stars reflect an evolutionary path different from that followed by solar neighbourhood stars (see Sect. 4.3); however, chemical separation may have changed their original composition. In particular, atomic diffusion could lower the sur- face ${ }^{7} \mathrm{Li}$ abundance inherited at birth (Charbonnel et al. 2021). We note that the star with the highest ${ }^{7} \mathrm{Li}$ abundance in our sample, $A(\mathrm{Li})=3.54 \pm 0.06 \mathrm{dex}$, and with $[\mathrm{Fe} / \mathrm{H}]=0.05 \pm 0.06$ is relatively hot $\left(T_{\text {eff }}=6670 \pm 66 \mathrm{~K}\right)$ and has $\log g=4.0 \pm 0.17$ dex, that is, it falls outside the Li-dip region according to Fig. 3. It also has a $\operatorname{good} \mathrm{S} / \mathrm{N}$ of 113 and $v \sin \mathrm{i}=117.4 \pm 1.1$, which enhances the chances that we are seeing its original lithium content unaltered by mixing processes. In fact, as discussed by Charbonnel et al. (2021), high rotation rates would counteract atomic diffusion. The information on stellar rotation is thus very useful in that it provides hints on the possible role played by internal ${ }^{7} \mathrm{Li}$ depletion processes in warm, metal-rich main-sequence stars.

\subsection{Evolution of lithium in the solar vicinity}

In this section, we focus on solar vicinity targets (i.e. clusters and stars with galactocentric distances $7<R_{\mathrm{GC}} / \mathrm{kpc}<9$ ). In Fig. 6 we compare the predictions of the parallel GCE model to ${ }^{7} \mathrm{Li}$ abundances of field stars and to average maximum ${ }^{7} \mathrm{Li}$ abundances of OCs selected from GES iDR6 (see Sect. 2). We assumed either the Spite plateau value, $A(\mathrm{Li})_{\mathrm{P}}^{\mathrm{obs}} \simeq 2.2 \mathrm{dex}$, or the BBN-predicted value, $A(\mathrm{Li})_{\mathrm{P}}^{\text {th }} \simeq 2.7 \mathrm{dex}$, as the initial ${ }^{7} \mathrm{Li}$ abundance in the GCE model. The contributions to ${ }^{7} \mathrm{Li}$ synthesis from RGB and AGB stars ${ }^{10}$, nova systems, and GCRs were included in all models, following the prescriptions outlined in Sect. 3.2. Lithium production through the $v$-process in massive stars was also implemented in one case (pink curves in Fig. 6).

The evolution of the thick-disc component is shown in the left panel, where the $\mathrm{x}$-axis reads, as usual, as a temporal axis. Because of the assumed fast evolution (see Grisoni et al. 2017), coupled to negligible production of ${ }^{7} \mathrm{Li}$ from both massive AGB stars (Ventura et al. 2000, 2020) and GCRs (Romano et al. 2001; Prantzos 2012) at the relevant metallicities, the abundance of ${ }^{7} \mathrm{Li}$ is predicted to remain almost flat during most of the thick-disc evolution (see also Guiglion et al. 2016; Grisoni et al. 2019).

${ }_{10}$ Possible production or destruction of ${ }^{7} \mathrm{Li}$ due to extra mixing pro-
cesses, such as thermohaline mixing in low-mass, bright RGB stars and
stellar rotation (Charbonnel et al. 2020), are not included in the adopted stellar models and yields (Ventura et al. 2013, 2020). 

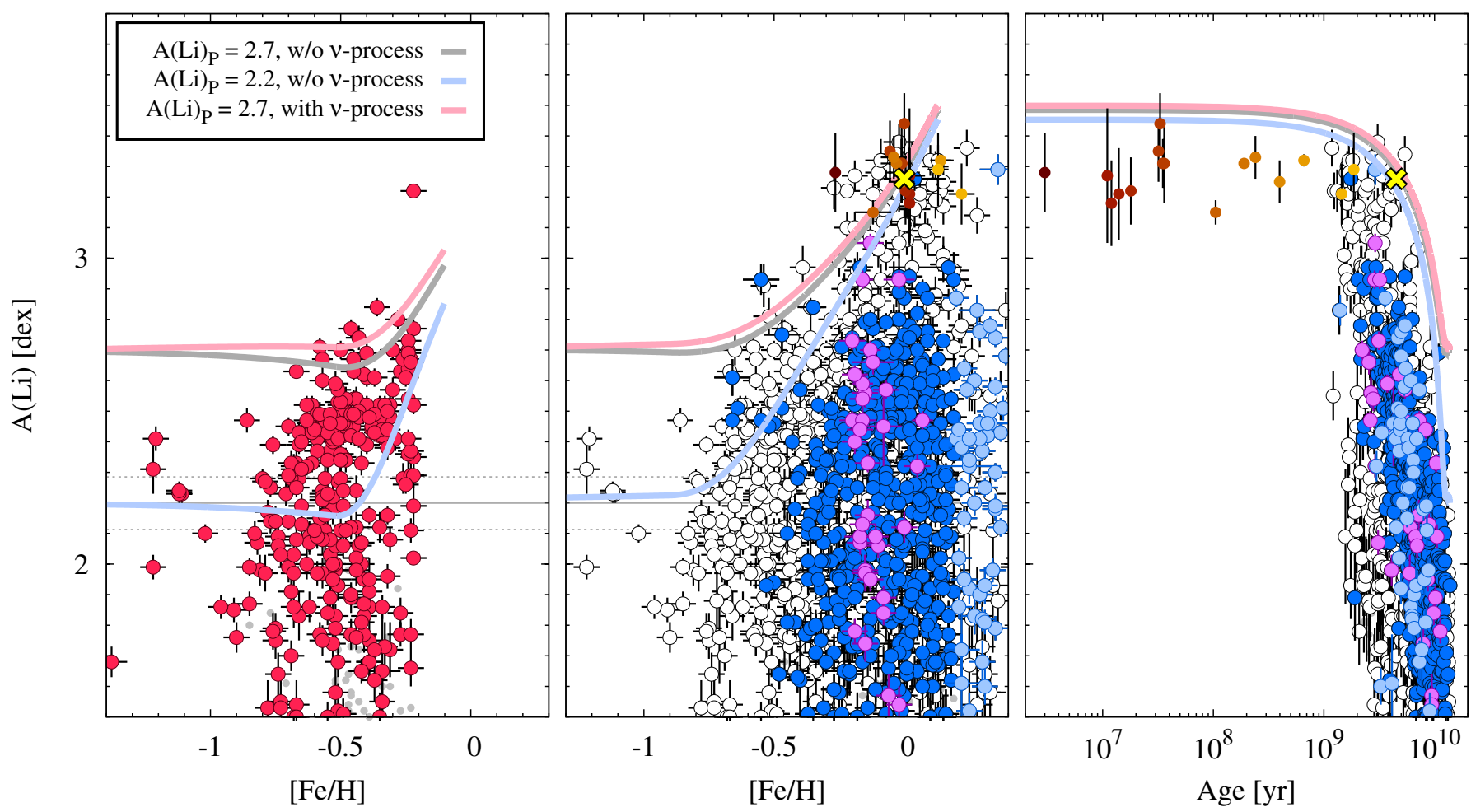

Fig. 6. Behaviour of $A(\mathrm{Li})$ as a function of $[\mathrm{Fe} / \mathrm{H}]$ (left and middle panels) and age (right panel) in the solar neighbourhood, for the thick- (left) and thin-disc components (middle and right panels). Lithium measurements for high- and low- $\alpha$ field stars in GES iDR6 are shown (red and blue circles, respectively), together with upper limits (grey dots). Cluster data are shown with brown shades for the younger clusters and yellow tones for the older ones. Only clusters and stars with $7<R_{\mathrm{GC}} / \mathrm{kpc}<9$ are considered for the comparison with the solar neighbourhood models. The meteoritic abundance from Lodders et al. (2009) is indicated with the multiplication sign. The predictions of the parallel GCE model are highlighted with different colours depending on the assumptions made about ${ }^{7} \mathrm{Li}$ synthesis and cosmological lithium abundance. We also show data for metal-rich $\alpha$-rich stars (magenta circles) and super metal-rich solar- $\alpha$ stars (light blue circles) found in the solar neighbourhood. These are not to be compared with the predictions of solar neighbourhood models and are used only for discussion purposes (see text). Empty circles are stars that lack an $[\alpha / \mathrm{Fe}]$ determination in GES iDR6; we assign most of them to the thin-disc component, based on their kinematics (see Sect. 4.5).

Actually, owing to the relatively high star formation efficiency, a mild decrease is expected due to stellar astration, until novae start releasing large amounts of ${ }^{7} \mathrm{Li}$ through their outbursts, that is, 1-2 Gyr from the onset of star formation (see Romano et al. 1999, their Fig. 3). This decrease is prevented if another short-lived (but still controversial) ${ }^{7} \mathrm{Li}$ factory is considered. The upper (pink) curve that does not bend downwards for $-1 \leq[\mathrm{Fe} / \mathrm{H}] / \mathrm{dex} \leq-0.5$ refers to a model implementing the contribution from core-collapse $\mathrm{SNe}$ that produce ${ }^{7} \mathrm{Li}$ in neutrino-induced reactions in the $\mathrm{He}$ and $\mathrm{C}$ shells (Domogatskii et al. 1978; Woosley et al. 1990). Starting from a primordial high ${ }^{7} \mathrm{Li}$ abundance, in accordance with SBBN predictions, and considering all possible ${ }^{7} \mathrm{Li}$ sources, the model reproduces reasonably well the upper envelope of the GES iDR6 ${ }^{7} \mathrm{Li}$ measurements for thick-disc members. On the contrary, a model starting from the Spite plateau value cannot explain the ${ }^{7} \mathrm{Li}$ abundances measured in thick-disc stars in the metallicity range $-0.8 \leq[\mathrm{Fe} / \mathrm{H}] / \mathrm{dex} \leq-0.3$. It should be noted, though, that candidate thick-disc stars were selected via chemical selection criteria (see Sect. 4.1). We will come back to this issue in Sect. 4.5. It is also worth noticing that, although the model attains ISM $A(\mathrm{Li})$ values as high as $\sim 3.0$ dex, very few stars form with these high ${ }^{7} \mathrm{Li}$ abundances, because of the low-level star formation activity that characterises the thick disc during its latest evolutionary stages.

The middle panel of Fig. 6 is analogous to the left panel, but refers to the thin-disc component. The blue circles in this plot are stars that were assigned to the thin-disc component after a chemical selection based on their metallicities and $[\alpha / \mathrm{Fe}]$ ratios. Metal-rich $\alpha$-rich stars and super metal-rich solar- $\alpha$ stars found in the solar vicinity today (magenta and light blue circles, respectively) are likely migrating from inner Galactic regions (Guiglion et al. 2019, and references therein) and, as such, reflect different chemical enrichment paths. Therefore, one should not compare their abundances to the predictions of solar neighbourhood models (see also Guiglion et al. 2019). At variance with the results for the thick-disc component, none of our theoretical curves for the local thin disc is found to fold downwards in the $A(\mathrm{Li})-[\mathrm{Fe} / \mathrm{H}]$ plane. This is due to the less efficient star formation assumed for the thin disc (see Grisoni et al. 2017) coupled to the continuous infall of gas of primordial chemical composition that replenishes the ISM with fresh ${ }^{7} \mathrm{Li}$ on long timescales. Owing to the less efficient star formation and longer infall timescale, the 'reverse knee' indicating the onset of significant ${ }^{7} \mathrm{Li}$ production from novae in the lithium tracks occurs at lower metallicities than in the thick disc, around $[\mathrm{Fe} / \mathrm{H}] \simeq-0.7$ dex or even lower, depending on the value assumed for $A(\mathrm{Li})_{\mathrm{P}}$. Again, the upper envelope of the observations is best explained by assuming $A(\mathrm{Li})_{\mathrm{P}}^{\text {th }} \simeq 2.7 \mathrm{dex}$.

In recent years, high-resolution spectroscopic surveys have identified a decreasing trend of the lithium content in local field dwarfs at super-solar metallicities (Delgado Mena et al. 2015; Guiglion et al. 2016; Bensby \& Lind 2018; Fu et al. 2018). Such a puzzling behaviour has called for some ad hoc reduc- 


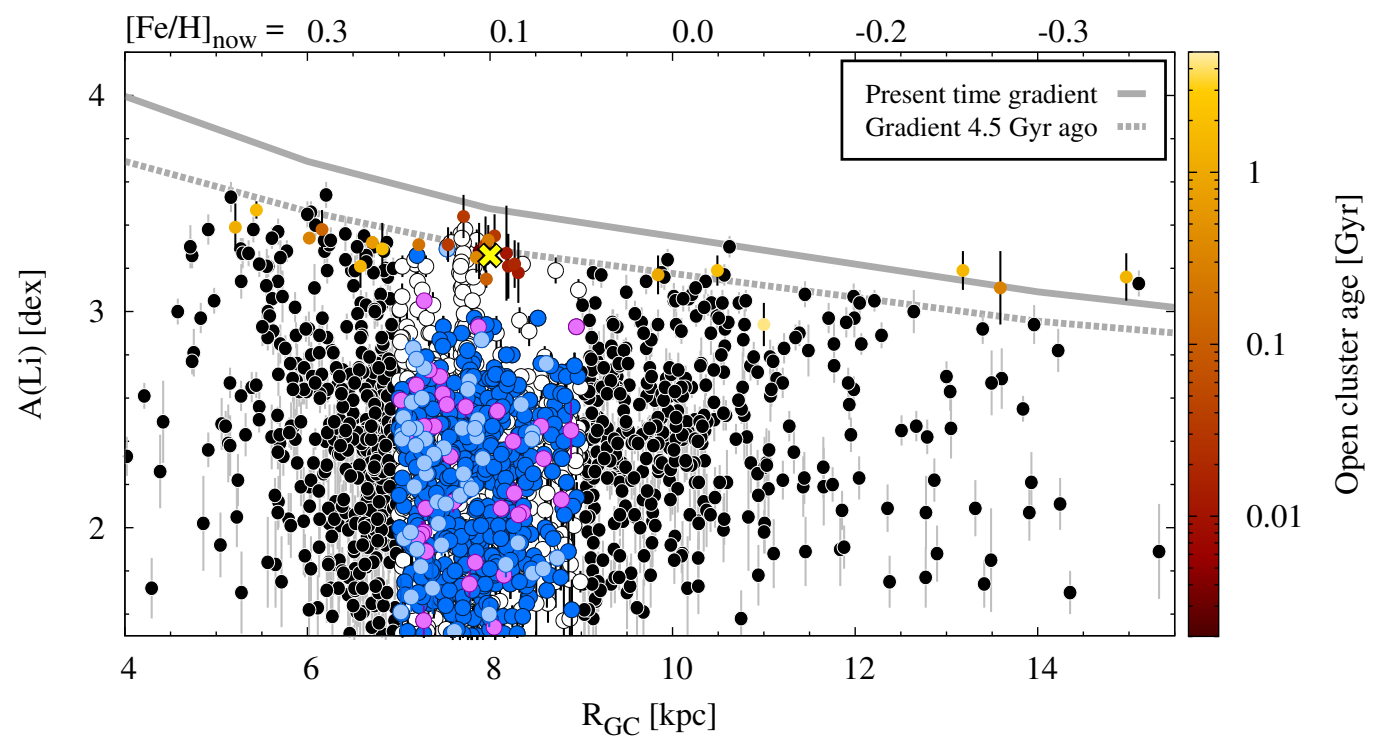

Fig. 7. Radial profiles of lithium abundance. The theoretical predictions refer to the gradient at the Sun's birth $(t=9.2$ Gyr, dotted curve $)$ and at the current time ( $t=13.7 \mathrm{Gyr}$, solid line). Data for our sample of GES iDR6 field stars, chemically separated into different sub-populations only in the solar neighbourhood region, are shown in different colours. Blue, magenta, and light blue circles identify, respectively, low- $\alpha$, metal-rich $\alpha$-rich, and metal-rich solar- $\alpha$ stars. Black circles refer to stars that inhabit either the inner or the outer disc. Average maximum ${ }^{7} \mathrm{Li}$ abundances for 26 OCs selected from GES iDR6 are also displayed (small colour-coded circles). The meteoritic abundance from Lodders et al. (2009) is indicated with the multiplication sign. On the top x-axis we report the current metallicity of the ISM as predicted by the model at different radii.

tion of the stellar yields or contamination from thick-disc stars (Prantzos et al. 2017). Alternatively, a drop in the nova outburst rate at high metallicities, which would lower the total lithium production from these binary systems (Fu et al. 2018; Grisoni et al. 2019), or radial migration, bringing relatively old and cool metal-rich thin-disc stars that have largely depleted their original ${ }^{7} \mathrm{Li}$ from inner radii to the solar neighbourhood (Guiglion et al. 2019), have been suggested. Regarding the last point, we note that, indeed, the vast majority of the stars labelled as super metal-rich, solar- $\alpha$ stars in our sample are older than 3-4 Gyr and cooler than $6000 \mathrm{~K}$ (light blue circles in Fig. 6, right panel, and Fig. 4, respectively), which makes them highly susceptible to internal lithium destruction processes.

Randich et al. (2020), based on GES iDR5, have shown that the average maximum ${ }^{7} \mathrm{Li}$ abundances of young, metal-rich OCs in the solar neighbourhood tend to cluster around the meteoritic value; the most metal-rich clusters found in the inner Galaxy display even higher abundances, $A(\mathrm{Li})>3.4$ dex. We confirm those findings, which in turn support the conclusions of AnthonyTwarog et al. (2018) and Guiglion et al. (2019): the observed decrease in $A(\mathrm{Li})$ for super-solar metallicities is not real, but caused by sample selection effects (see also Charbonnel et al. 2021). The lithium abundance in the local ISM traced by young OCs and warm field dwarfs in our sample is not decreasing; rather, it seems to flatten. However, we cannot exclude an increase in the last $4.5 \mathrm{Gyr}$, due to possible effects of atomic diffusion on warm, metal-rich F-type dwarfs that call for some caution when interpreting the metal-rich data (Charbonnel et al. 2021).

From the middle panel of Fig. 6 it appears also that the GCE model underestimates the ${ }^{7} \mathrm{Li}$ content of the ISM in the $-0.5 \leq[\mathrm{Fe} / \mathrm{H}] / \mathrm{dex}<0.0$ metallicity range. The culprit for this mismatch may be the simplifying hypotheses made to implement nova nucleosynthesis in the GCE code that lead to a monotonic increase in the ${ }^{7} \mathrm{Li}$ abundance in time since the first novae start to pollute the ISM. A reduction of the nova outburst rate at high metallicities, as suggested by Fu et al. (2018) and Grisoni et al. (2019), may be needed (and is supported by independent obser- vational evidence; see Gao et al. 2014, 2017; Yuan et al. 2015). A larger ${ }^{7} \mathrm{Li}$ production (potentially from novae) in the metallicity range $-0.5 \leq[\mathrm{Fe} / \mathrm{H}]<0.0$ is, instead, necessary to fit the data. In order to better assess these points, we analyse the implications of a radial ${ }^{7} \mathrm{Li}$ abundance gradient in the next section.

\subsection{The Galactic lithium gradient}

We followed the evolution of the abundance of ${ }^{7} \mathrm{Li}$ in the ISM at different positions across the thin disc by using the prescriptions of model 1 IM B by Grisoni et al. (2018). This model assumes the inside-out scenario for disc formation (see Chiappini et al. 2001, and references therein) and a variable star formation efficiency along the disc. We refer the reader interested in details to the original publications. Here it will suffice to say that the model has been carefully calibrated against a set of tight observational constraints ${ }^{11}$, and that we did not make any attempt to fine-tune the model parameters in order to improve the agreement with the ${ }^{7} \mathrm{Li}$ data. The ages of the OCs were taken from Cantat-Gaudin et al. (2020, see Table 2), while those of the field stars were estimated using the aussieq 2 tool (see Sect. 2.1 and Casali et al. 2020). The galactocentric distances were homogeneously computed using astropy (see Sect. 2.2).

The theoretical gradients at different ages (now and 4.5 Gyr ago) are compared to the data in Fig. 7. The model used to compute the gradients starts from the SBBN-predicted primordial ${ }^{7} \mathrm{Li}$ abundance and does not take lithium production from the $v$-process into account. Unsurprisingly, the large majority of the field stars lie well below the model predictions. This can be readily understood as an age-temperature-rotation effect: only a minority of the stars in our sample, in fact, are relatively young (ages $<2$ Gyr), have $T_{\text {eff }}$ in excess of $6800 \mathrm{~K}$ and display high

These include the present-time gradients of several chemical ele-
ments along the disc and their evolution, as well as the radial profiles
of gas, stars, and star formation rate (see Chiappini et al. 2001, for a detailed discussion). 

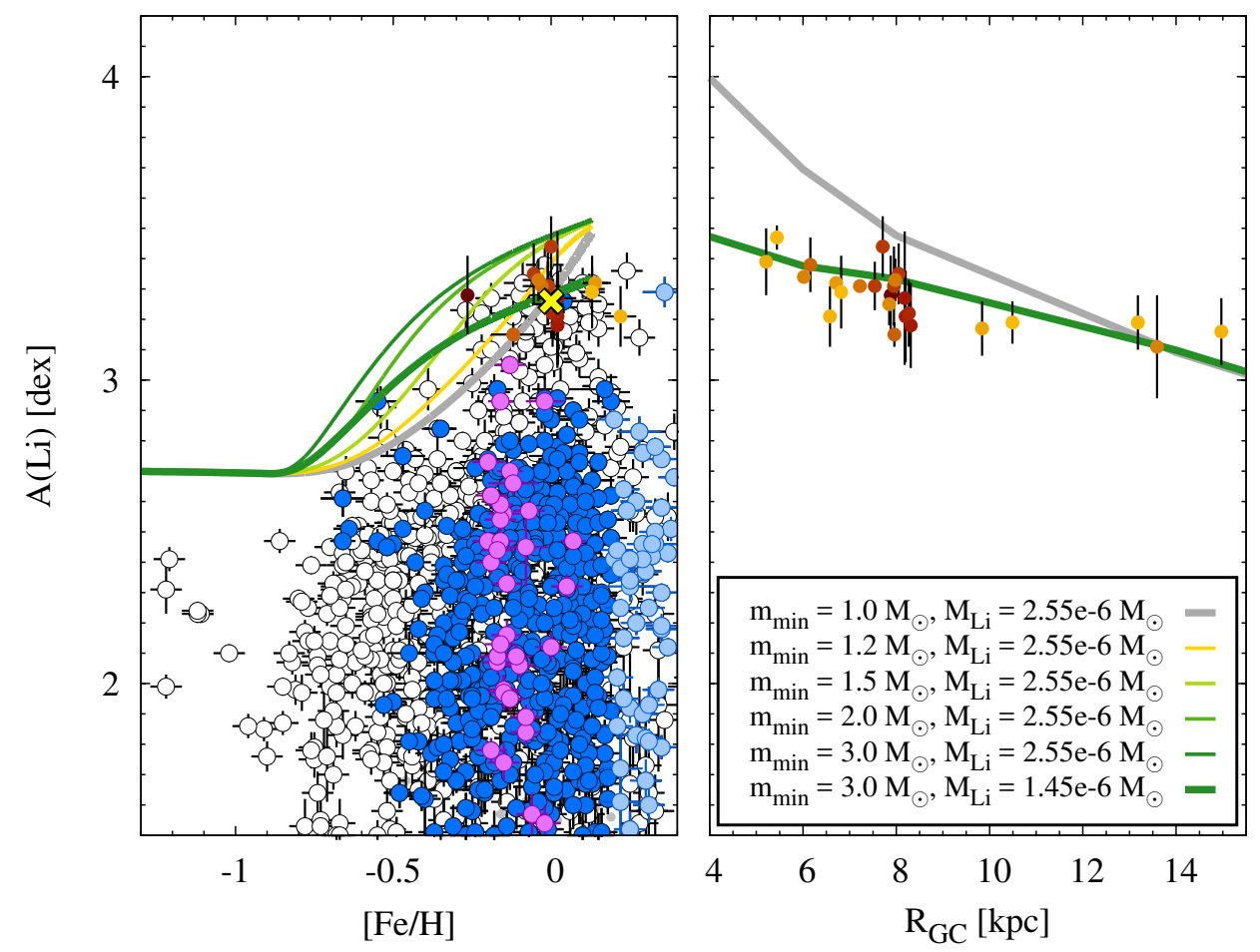

Fig. 8. Behaviour of $A(\mathrm{Li})$ as a function of $[\mathrm{Fe} / \mathrm{H}]$ in the local thin disc $\left(R_{\mathrm{GC}}=7-9 \mathrm{kpc}\right.$, left panel $)$ and as a function of the galactocentric distance at the present time (right panel). Lithium measurements for field low- $\alpha$, mr $\alpha$ r, and super metal-rich solar- $\alpha$ stars from GES iDR6 are shown (blue, magenta, and light blue circles, respectively, left panel only) together with data for OCs colour-coded according to the cluster's age (see the vertical bar on the right in Fig. 7). Only clusters with ages $<2$ Gyr are shown. The predictions of our fiducial GCE model are shown as thick grey lines. The thin solid lines illustrate the effects of reducing the mass range of primary stars entering the formation of nova systems (left panel only) while keeping the predicted current nova outburst rate fixed. The thick green lines refer to a model in which the nova yield (i.e. the total amount of ${ }^{7} \mathrm{Li}$ ejected by a typical nova during its lifetime, dubbed $M_{\mathrm{Li}}$ in the legend) is also reduced. See text for details.

rotation rates (see Figs. 2 and 5), which are the right characteristics to possibly show up as un-depleted stars (see Sect. 4.1; see also Gao et al. 2020; Charbonnel et al. 2021). The OCs span a much narrower age range, from $3 \mathrm{Myr}$ to about $2 \mathrm{Gyr}$, with only one cluster (NGC 2243, at $R_{\mathrm{GC}}=11 \mathrm{kpc}$ ) being older than 4 Gyr. We retained this cluster to increase the statistics in the outer disc. Its average maximum lithium abundance was computed using 7 post turn-off members with $T_{\text {eff }}$ between $6064 \mathrm{~K}$ and $6314 \mathrm{~K}$, in an effort to minimise the effects of ${ }^{7} \mathrm{Li}$ depletion. This notwithstanding, NGC 2243 shows a ${ }^{7} \mathrm{Li}$ abundance $\sim 0.2-0.25$ dex lower than those of the other outer disc clusters. While the low ${ }^{7} \mathrm{Li}$ content of NGC 2243 could be partly due to depletion as its stars are ageing, it could also be partly due to a lower initial ${ }^{7} \mathrm{Li}$ abundance inherited from the ISM when the cluster formed $4.4 \mathrm{Gyr}$ ago. Indeed, the GCE model predicts an increase of $0.3-0.1$ dex in the ISM ${ }^{7} \mathrm{Li}$ abundance across the disc in the last $4.5 \mathrm{Gyr}$ (cfr. the dashed and solid lines in Fig. 7). The metallicity of NGC 2243, $[\mathrm{Fe} / \mathrm{H}]=-0.44 \pm 0.05 \mathrm{dex}$, is lower than the solar one and lower (by $\sim 0.2-0.4$ dex) than those of the other outer disc clusters, which would exclude an inner Galaxy origin and favour the evolutive picture.

For $R_{\mathrm{GC}} \leq 10 \mathrm{kpc}, \mathrm{OCs}$ and warm field dwarfs trace the same ${ }^{7} \mathrm{Li}$ abundance gradient. At larger galactocentric distances, the gradient seems to flatten according to the OC data, while $A(\mathrm{Li})$ keeps decreasing in field stars, though it should be noted that the statistics in the outer disc are very poor. Taken at face value, the data seem to suggest that the observed gradient is flatter than the one predicted by our fiducial model. In particular, the data for the inner Galaxy $\left(R_{\mathrm{GC}} \leq 8 \mathrm{kpc}\right)$ agree very well with the predictions of the model at $4.5 \mathrm{Gyr}$ ago. A possible explanation is that high- metallicity clusters and field stars in the inner disc were born with a higher lithium abundance, perhaps not too far from that predicted by the model at the present time, $A(\mathrm{Li}) \simeq 3.5-3.8 \mathrm{dex}$, and show superficial abundances altered by depletion or atomic diffusion (see Charbonnel et al. 2021). Alternatively, we are seeing unaltered ${ }^{7} \mathrm{Li}$ abundances and the resulting mild inner disc gradient points to the need for a reduction of lithium production at super-solar metallicities. This, in the framework of our model, can be interpreted as evidence in favour of the hypothesis that the nova outburst rate is reduced at high metallicities (Fu et al. 2018; Grisoni et al. 2019, and references therein). On the other hand, the predicted present-time gradient agrees very well with the OC data in the outer disc. These external regions have not reached solar metallicities yet and, as such, they have not entered the regime in which a downward correction of the nova outburst rate could be required. Thus, a model implementing a metallicity dependence of the nova progenitor formation rate, as proposed by Grisoni et al. (2019), offers a viable explanation for the whole set of homogeneous ${ }^{7} \mathrm{Li}$ abundances examined in this paper. Another possible explanation is considered in the following paragraphs.

\subsection{An alternative model}

In our fiducial model, the star formation rate in the solar vicinity declines slowly in time after an early period of intense activity. The nova outburst rate, however, keeps always increasing mildly in time (see Romano et al. 1999, their Fig. 3), due to the contribution of the numerous lowest-mass stars $\left(m \sim 1 \mathrm{M}_{\odot}\right)$ that enter the formation of nova systems with lifetimes of the order of the 


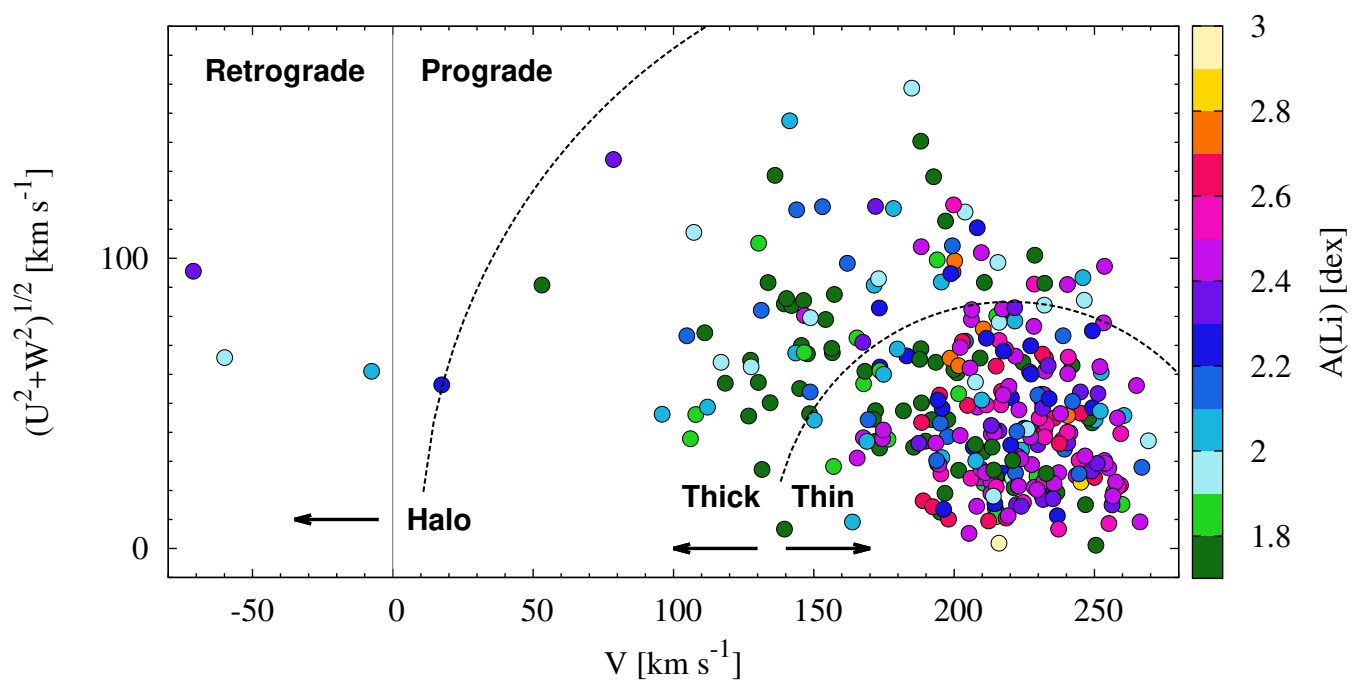

Fig. 9. Toomre diagram (LSR-corrected) for our sub-sample of high- $\alpha$ stars, colour-coded according to their ${ }^{7} \mathrm{Li}$ abundance (see vertical bar on the right). Constant total space velocities with respect to the LSR of $V_{\text {tot }}=85$ and $210 \mathrm{~km} \mathrm{~s}^{-1}$ are marked with dashed curves. Low- $\alpha$, metal-rich $\alpha$-rich, and super metal-rich solar- $\alpha$ stars (not shown to avoid overcrowding) are consistent with thin-disc kinematics.

age of the Universe (see e.g. Romano et al. 2005, their Fig. 3). If the impact of such long-lived stars is lowered, one can expect a nova outburst rate tracking more closely the star formation rate and, hence, a lower ${ }^{7} \mathrm{Li}$ production at late times.

In Fig. 8, left panel, we show the effects of reducing the mass range of the primary stars that enter the formation of nova systems on the $A(\mathrm{Li})$ versus $[\mathrm{Fe} / \mathrm{H}]$ trend predicted for the local thin disc. The fiducial model, where the minimum mass of the stars entering nova systems is set to $1 \mathrm{M}_{\odot}$, predicts the steepest increase in the lithium abundance from the SBBN-predicted value to the meteoritic one. As the minimum mass increases, the rise off the plateau is found to occur at lower metallicities and the late evolution is characterised by flatter slopes (thin lines from yellow to dark green in Fig. 8, left panel).

All the models are calibrated to obtain the same current nova outburst rate (i.e. 17 events $\mathrm{yr}^{-1}$; Izzo et al. 2015, and references therein). This makes the revised models overestimate the ${ }^{7} \mathrm{Li}$ abundance observed in meteorites. The thick green line in Fig. 8, left panel, shows the predictions of a model (hereinafter dubbed alternative model) where both the mass range of WD progenitors entering the formation of nova systems and the average ${ }^{7} \mathrm{Li}$ yield per nova are reduced so as to optimally fit the local data within the errors. Of course, a strong degeneracy affects the relevant model parameters and the one proposed here is not a unique choice. A thorough investigation of the parameter space, however, is beyond the scope of the present study and is deferred to a future paper.

The right panel of Fig. 8 displays the present-day gradients of $A(\mathrm{Li})$ across the Milky Way disc predicted by the fiducial and alternative models (grey and green thick lines, respectively), in comparison to our OC data excluding the sole cluster with age $>2$ Gyr (NGC 2243). Lacking the contribution of the longlived stars in the $1-3 \mathrm{M}_{\odot}$ mass range, the alternative model predicts a gradient much flatter than that expected according to the fiducial model. This milder theoretical gradient agrees very well with the observed one traced by the average maximum ${ }^{7} \mathrm{Li}$ abundances of the selected OCs (see Sect. 2.1), though one should always keep in mind the caveats spelled out in the previous sections. In particular: (i) the OC data include stars on the cool side of the Li dip, thus, it is possible that these have suffered some pre-main-sequence or main-sequence depletion (in which case the depletion is metallicity dependent); (ii) higher metallicity means a thicker sub-photospheric convection zone, hence, the OCs nearer the Galactic centre, being more metal-rich, may have depleted their Li from a higher level than the more metalpoor clusters further out in the disc and this would have the effect of flattening the initial gradient; (iii) the effects of atomic diffusion on the shape of the inner gradient still need to be clarified. Therefore, at present we cannot discard a steeper gradient - any lying in between the green and the grey curves in Fig. 8, right panel.

\subsection{Sanity check on membership and accreted stars}

In Sect. 4.1 we adopt the chemical selection criteria defined by Recio-Blanco et al. (2014) and used in lithium studies by Guiglion et al. (2016, 2019) and Fu et al. (2018) as an option to separate thick-disc stars (identified as high- $\alpha$ stars) from thindisc ones (identified as low- $\alpha$ stars). The adoption of different selection criteria, however, leads to different samples of candidate thick- and thin-disc stars (e.g. Bensby et al. 2003, 2014; Franchini et al. 2020). In particular, with regard to ${ }^{7} \mathrm{Li}$ evolution, Bensby \& Lind (2018) caution that the chemical selection may cause a contamination of the thick-disc sample with thin-disc members, especially at higher metallicities, which may lead to a spurious trend of increasing ${ }^{7} \mathrm{Li}$ abundance with metallicity for the thick disc.

In Fig. 9 we show the LSR-corrected (assuming $V_{\mathrm{LSR}}=220$ $\mathrm{km} \mathrm{s}^{-1}$ ) velocity components for our sub-sample of high- $\alpha$ stars in a Toomre diagram. If a kinematical selection is applied, only 25 per cent of the high- $\alpha$ stars are classified as thick-disc members; in particular, the stars with the highest ${ }^{7} \mathrm{Li}$ abundances are all thin-disc members (in agreement with the results of Bensby \& Lind 2018, based on a different sample of stars). Moreover, if a cut in age is applied, so that only stars older than 8 Gyr are retained, no thick-disc stars are left in the diagram with $A(\mathrm{Li})>$ 2.5 dex.

The velocity space is a useful tool to pinpoint the origin of stars, especially when coherent moving groups can be identified 


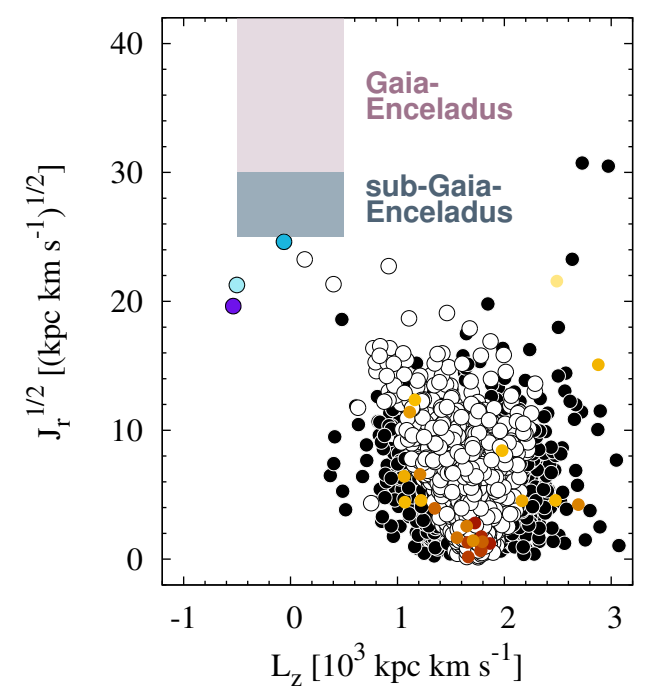

Fig. 10. $\sqrt{J_{r}}$ versus $L_{z}$ action space for our complete sample. Empty circles are disc stars in the solar vicinity, full black circles indicate targets observed at $R_{\mathrm{GC}} \leq 7 \mathrm{kpc}$ or $R_{\mathrm{GC}} \geq 9 \mathrm{kpc}$, and circles in shades from yellow to maroon are OCs. The three retrograde halo stars are colour-coded according to their ${ }^{7} \mathrm{Li}$ content (see Fig. 9). The loci of Gaia-Enceladus and sub-Gaia-Enceladus stars (see Feuillet et al. 2020) are highlighted.

(see Helmi 2020, for a recent review). We find that our sample includes 3 retrograde (counter-rotating) halo stars. We analyse their position in a diagram of angular momentum $L_{z}$ versus square root of radial action in Fig. 10. Feuillet et al. (2020) have pointed out that Gaia-Enceladus stars are found in a restricted region of this space, $30 \leq \sqrt{J_{r}} /\left(\mathrm{kpc} \mathrm{km} \mathrm{s}^{-1}\right) \leq 50$ and $-500 \leq$ $L_{z} /\left(\mathrm{kpc} \mathrm{km} \mathrm{s}^{-1}\right) \leq 500$ (clean sample); accreted stars may also be found at slightly lower $\sqrt{J_{r}}$ values (sub-Gaia-Enceladus sample). One of our retrograde halo stars is marginally compatible with being a member of the latter sample. Clearly, a full chemical tagging may shed more light on an accreted origin of our retrograde stars, but this is beyond the scope of the present paper. We note, however, that all our retrograde stars have $A(\mathrm{Li})$ values consistent with the Spite plateau level (see also Molaro et al. 2020a) and, hence, with no ${ }^{7} \mathrm{Li}$ enrichment as expected for stars hosted in dwarf galaxies according to Matteucci et al. (2021). Those authors compute detailed chemical evolution models for several dwarf spheroidal and ultra-faint dwarf galaxies and show that, if most ${ }^{7} \mathrm{Li}$ is forged in nova outbursts, as also assumed in this paper, its abundance in Milky Way dwarf satellites must lie flat at the Spite plateau level; only a few systems experiencing a more prolonged star formation activity may host relatively metal-rich stars that display higher lithium abundances.

All our retrograde stars have $T_{\text {eff }}<6000 \mathrm{~K}$, namely, they fall on the cool side of the dip. Measurements of ${ }^{7} \mathrm{Li}$ abundances in accreted stars on the warm side of the dip would be of the utmost importance to characterise the evolution of ${ }^{7} \mathrm{Li}$ in their galactic progenitors, since they would possibly reflect un-depleted abundances. Such stars must be rare - because of stellar evolution effects combined with the expected old ages - but some relatively young objects gained through recent mergers could still be found in large spectroscopic surveys targeting larger stellar samples than GES, such as GALAH (De Silva et al. 2015), or WEAVE (Dalton et al. 2012) and 4MOST (de Jong et al. 2012) in the future.

\section{Discussion}

Nowadays large spectroscopic surveys can secure precise radial velocities and homogeneous abundances for light and heavy chemical elements, for as many as hundreds of thousands of stars. This information, coupled to accurate parallaxes and proper motions measured by the European Space Agency Gaia mission, provides unprecedented constraints to GCE models. In particular, when dealing with the Galactic evolution of lithium the powerfulness of modern surveys is apparent. We can now select stars that belong to different Galactic components and analyse how ${ }^{7} \mathrm{Li}$ enrichment proceeded in different substructures, including regions of the disc located at different galactocentric distances to trace the gradient. We can exclude contaminants, such as stars that migrated from the inner disc, from local samples and get a cleaner picture of the evolutionary paths followed by coherent groups of stars, rather than being dealing with the superposition of different evolutionary sequences (see Guiglion et al. 2019, for a first discussion on this aspect). As regards this point, it is worth stressing that we identified inner disc intruders in our sub-sample of solar neighbourhood stars via chemical selection criteria and that we based our main conclusions on objects - both clusters and field stars - that are younger than $2 \mathrm{Gyr}$ and, hence, did not travel significant distances across the disc (Anders et al. 2017; Casamiquela et al. 2017).

At present, the trends we see for ${ }^{7} \mathrm{Li}$ abundance as a function of metallicity, age, and galactocentric distance are still hampered by several uncertainties. In most cases, ${ }^{7} \mathrm{Li}$ abundances are computed using LTE plane-parallel atmospheres; non-LTE corrections calculated with $1 \mathrm{D}$ models are often applied, while $3 \mathrm{D}$ non-LTE corrections are considered rarely. High rotation rates may broaden the lines and lead to inaccurate results in case of automatic abundance derivation. The exact location of the lithium dip in dependence of stellar parameters such as age and metallicity is not well known and more OC studies are needed to sort out all the pieces (e.g. Anthony-Twarog et al. 2021; Randich \& Magrini 2021, and references therein). Last in order of time, Charbonnel et al. (2021) warn that the effects of atomic diffusion in warm metal-rich stars deserve further analysis before we can draw any firm conclusion on the actual behaviour of $A(\mathrm{Li})$ in the high-metallicity regime. This impacts both the local late Galactic evolution and the slope of the gradient in the inner disc.

However, the ${ }^{7} \mathrm{Li}$ abundance measured in meteorites provides a good anchor for the models, as it has been 'frozen' since the time of the solar system formation 4.5 Gyr ago. Our GCE model, assuming that most lithium is produced in nova outbursts, can explain the meteoritic lithium abundance and, as we show in this paper, is able to reproduce either a flatter or a steeper $A(\mathrm{Li})$ gradient, depending on the assumed mass range for the WD nova progenitors. Thus, determining the exact slope of the gradient is a crucial step towards a better understanding of lithium production on galactic scales.

Another important issue that has to be fixed is that of the actual nova outburst rate. Recently, De et al. (2021) have suggested that a large population of highly obscured novae have been systematically missed in optical searches. The current nova outburst rate would be $46.0 \pm 12.5$ events $\mathrm{yr}^{-1}$, larger than the estimate of 17 events $\mathrm{yr}^{-1}$ assumed in this work (see also Izzo et al. 2015). This would point to a lower average ${ }^{7} \mathrm{Li}$ yield from novae, in better agreement with the results of the majority of hydrodynamical simulations of the outburst. 


\section{Conclusions}

Using spectroscopic data from the last internal data release of the GES and astrometric information from Gaia EDR3, we have built a catalogue of 3210 stars in the Milky Way field that have (i) high-precision $1 \mathrm{D} \mathrm{LTE}{ }^{7} \mathrm{Li}$ abundances or upper limits, (ii) homogeneous stellar parameters and (iii) $[\alpha / \mathrm{Fe}]$ ratios (for a subsample of stars), (iv) stellar ages, (v) galactocentric distances, (vi) space velocity components, and (vii) orbital parameters. The field star sample is complemented with estimates of the average maximum ${ }^{7} \mathrm{Li}$ abundances for 26 star forming regions and OCs for which GES iDR6 delivers homogeneous stellar parameters and abundances of confirmed members. All clusters, bar one, have ages $<2$ Gyr. After a careful inspection of the data, we conclude that:

- In the metallicity range $-0.3<[\mathrm{Fe} / \mathrm{H}] / \mathrm{dex}<+0.3$, where our GES iDR6 field and OC samples overlap, field and cluster pre-main-sequence, main-sequence, and turn-off stars trace the same evolution of lithium. This is due to the fact that the last internal data release of the GES includes field stars with ages in the range 1-2 Gyr that were absent in previous releases of the survey (see Thompson et al. 2018). If only field stars older than 2 Gyr are considered, the average maximum ${ }^{7} \mathrm{Li}$ abundances estimated for the OCs lie systematically above the upper envelope of the field star measurements. Field stars older than 2 Gyr, in fact, fall in the lithium dip region or on the cool side of the dip, where their ${ }^{7} \mathrm{Li}$ abundances can be affected by various degrees of depletion.

- The average maximum ${ }^{7} \mathrm{Li}$ abundances of most OCs agree well with the abundance of lithium measured in meteorites that have not suffered any depletion.

- Our OC sample spans a wide range of galactocentric distances, $5 \leq R_{\mathrm{GC}} / \mathrm{kpc}<15$. The current ${ }^{7} \mathrm{Li}$ gradient traced by OCs is a mild one, ranging from $A(\mathrm{Li}) \sim 3.4$ dex in the inner Galaxy to $A(\mathrm{Li}) \sim 3.1$ dex in the outer disc. This gradient is also supported by observations of warm field stars. It is unclear at present whether atomic diffusion or other effects steepen the gradient in the inner Galaxy.

- We have discovered three counter-rotating stars in our field star sample that may be accreted stars. Their ${ }^{7} \mathrm{Li}$ abundances are consistent with the Spite plateau value, $A(\mathrm{Li})_{\mathrm{P}}^{\mathrm{obs}}=$ $2.199 \pm 0.086$ (Sbordone et al. 2010).

Recently, Grisoni et al. (2019) applied the parallel GCE model to the study of the evolution of ${ }^{7} \mathrm{Li}$ in the Galactic thick and thin discs. However, their analysis was limited to the solar vicinity. Here, we take advantage of the homogeneous dataset secured by GES iDR6 for field and OC stars to better assess the evolution of the abundance of ${ }^{7} \mathrm{Li}$ at high metallicities in the solar neighbourhood, as well as its gradient across the Milky Way disc. We run a fiducial model, where nearly 2 per cent of the stars with initial mass in the range $1-8 \mathrm{M}_{\odot}$ enter the formation of nova systems as primary stars, and each nova is assumed to eject $M_{\mathrm{Li} \text {, tot }}^{\text {nova }}=2.55 \times 10^{-6} \mathrm{M}_{\odot}$ during its lifetime (Romano et al. 1999; Izzo et al. 2015). We also run an alternate model, where 16 per cent of the stars in the $3-8 \mathrm{M}_{\odot}$ mass range are primary stars in binary systems that give rise to nova outbursts, and the average mass of ${ }^{7} \mathrm{Li}$ ejected in total by each nova is $M_{\mathrm{Li} \text {, tot }}^{\text {nova }}=$ $1.45 \times 10^{-6} \mathrm{M}_{\odot}$. We find that:

- The fiducial model underestimates the upper envelope of the observations as traced by both warm field stars and OCs in the solar neighbourhood, except for the super-solar metallicity regime, where the ${ }^{7} \mathrm{Li}$ abundances could be slightly overestimated. The fiducial model also seems to predict a present-time gradient steeper than the observed one. However, Charbonnel et al. (2021) caution that the possible effects of atomic diffusion on warm stars tracing the highmetallicity trends have not yet been fully understood. These could reconcile the model predictions with the observations. - The alternative model fits (by construction) the upper envelope of the local data in a $A(\mathrm{Li})-[\mathrm{Fe} / \mathrm{H}]$ plane and the current gradient along the disc.

- A model in which most ${ }^{7} \mathrm{Li}$ comes from nova outbursts explains the evolution of this fragile element well. However, the parameters introduced to implement the nova nucleosynthesis are highly degenerate and the proposed best-fitting model is, thus, not necessarily unique.

More data are needed to probe if novae are the main lithium factories and to assess whether their rate flattens out at late times. This latter requirement, in particular, might have implications for the masses of the WD nova progenitors, and it deserves further theoretical investigation. At present, stellar yields of single low-, intermediate-, and high-mass stars are too low for these stars to contribute sensibly to the evolution of ${ }^{7} \mathrm{Li}$ on galactic scales, apart perhaps from some contribution from the $v$-process in massive stars that would counterbalance the effects of stellar astration during early Galactic evolution. We also need to better understand the mechanisms of lithium depletion in stars and their dependences on stellar mass, metallicity, and other parameters, such as rotation. There is a long way to go, but it is certainly worthwhile.

Acknowledgements. This work is based on data products from observations made with ESO Telescopes at the La Silla Paranal Observatory under programme IDs 188.B-3002, 193.B-0936, and 197.B-1074. These data products have been processed by the Cambridge Astronomy Survey Unit (CASU) at the Institute of Astronomy, University of Cambridge, and by the FLAMES/UVES reduction team at INAF, Osservatorio Astrofisico di Arcetri. These data have been obtained from the Gaia-ESO Survey Data Archive, prepared and hosted by the Wide Field Astronomy Unit, Institute for Astronomy, University of Edinburgh, which is funded by the UK Science and Technology Facilities Council. This work was partly supported by the European Union FP7 programme through ERC grant number 320360 and by the Leverhulme Trust through grant RPG-2012-541. We acknowledge the support from INAF and from the Italian Ministry of Education, University and Research (Ministero dell'Istruzione, dell'Università e della Ricerca, MIUR) in the form of the grant Premiale VLT 2012. The results presented here benefit from discussions held during the GaiaESO workshops and conferences supported by the ESF (European Science Foundation) through the GREAT Research Network Programme. This work has made use of data from the European Space Agency (ESA) mission Gaia (https: //www. cosmos. esa.int/gaia), processed by the Gaia Data Processing and Analysis Consortium (DPAC, https://www.cosmos.esa.int/web/ gaia/dpac/consortium). Funding for the DPAC has been provided by national institutions, in particular the institutions participating in the Gaia Multilateral Agreement. LM, SR, GCas and AB acknowledges funding from MIUR Premiale 2016 MITiC. PB acknowledges support from the French National Research Agency (ANR) funded project "Pristine" (ANR-18-CE31-0017). VG acknowledges financial support at SISSA from the European Social Fund operational Programme 2014/2020 of the autonomous region Friuli Venezia Giulia. TB was funded by grant No. 2018-04857 from The Swedish Research Council. AJK acknowledges support from the Swedish National Space Agency (SNSA/Rymdstyrelsen). SLM is supported by funding from the Australian Research Council via Discovery Project DP180101791 and from the UNSW Scientia Fellowship program. FJE acknowledges financial support from the Spanish MINECO/FEDER through the grant AYA2017-84089 and MDM-2017-0737 at Centro de Astrobiología (CSIC-INTA), Unidad de Excelencia María de Maeztu, and from the European Union's Horizon 2020 research and innovation programme under Grant Agreement No. 824064 through the ESCAPE - The European Science Cluster of Astronomy and Particle Physics ESFRI Research Infrastructures project.

\section{References}

Adibekyan, V. Z., Santos, N. C., Sousa, S. G., \& Israelian, G. 2011, A\&A, 535, 
L11

Alonso, A., Arribas, S., \& Martínez-Roger, C. 1999, A\&AS, 140, 261

Anders, F., Chiappini, C., Rodrigues, T. S., et al. 2017, A\&A, 597, A30

Anthony-Twarog, B. J., Deliyannis, C. P., \& Twarog, B. A. 2021, AJ, 161, 159

Anthony-Twarog, B. J., Lee-Brown, D. B., Deliyannis, C. P., \& Twarog, B. A. 2018, AJ, 155, 138

Astropy Collaboration, Robitaille, T. P., Tollerud, E. J., et al. 2013, A\&A, 558, A33

Bailer-Jones, C. A. L., Rybizki, J., Fouesneau, M., Demleitner, M., \& Andrae, R. 2021, AJ, 161, 147

Bath, G. T. \& Shaviv, G. 1978, MNRAS, 183, 515

Bensby, T., Feltzing, S., \& Lundström, I. 2003, A\&A, 410, 527

Bensby, T., Feltzing, S., \& Oey, M. S. 2014, A\&A, 562, A71

Bensby, T. \& Lind, K. 2018, A\&A, 615, A151

Boesgaard, A. M. \& Tripicco, M. J. 1986, ApJ, 302, L49

Bonifacio, P. \& Molaro, P. 1997, MNRAS, 285, 847

Bouvier, J., Lanzafame, A. C., Venuti, L., et al. 2016, A\&A, 590, A78

Bovy, J. 2015, ApJS, 216, 29

Bovy, J., Allende Prieto, C., Beers, T. C., et al. 2012, ApJ, 759, 131

Cameron, A. G. W. 1955, ApJ, 121, 144

Cameron, A. G. W. \& Fowler, W. A. 1971, ApJ, 164, 111

Cantat-Gaudin, T., Anders, F., Castro-Ginard, A., et al. 2020, A\&A, 640, A1

Casali, G., Spina, L., Magrini, L., et al. 2020, A\&A, 639, A127

Casamiquela, L., Carrera, R., Blanco-Cuaresma, S., et al. 2017, MNRAS, 470, 4363

Cayrel, R. 1988, in The Impact of Very High S/N Spectroscopy on Stellar Physics, ed. G. Cayrel de Strobel \& M. Spite, Vol. 132, 345

Charbonnel, C., Borisov, S., de Laverny, P., \& Prantzos, N. 2021, A\&A, 649, L10

Charbonnel, C., Lagarde, N., Jasniewicz, G., et al. 2020, A\&A, 633, A34

Charbonnel, C. \& Primas, F. 2005, A\&A, 442, 961

Chiappini, C. 2009, in The Galaxy Disk in Cosmological Context, ed. J. Andersen, Nordströara, B. m, \& J. Bland-Hawthorn, Vol. 254, 191-196

Chiappini, C., Matteucci, F., \& Romano, D. 2001, ApJ, 554, 1044

Chugai, N. N. \& Kudryashov, A. D. 2020, Astronomy Letters, 46, 92

Clara, M. T. \& Martins, C. J. A. P. 2020, A\&A, 633, L11

Coc, A., Goriely, S., Xu, Y., Saimpert, M., \& Vangioni, E. 2012, ApJ, 744, 158

Dalton, G., Trager, S. C., Abrams, D. C., et al. 2012, in Society of Photo-Optical Instrumentation Engineers (SPIE) Conference Series, Vol. 8446, Groundbased and Airborne Instrumentation for Astronomy IV, ed. I. S. McLean, S. K Ramsay, \& H. Takami, 84460P

D’Antona, F. \& Matteucci, F. 1991, A\&A, 248, 62

De, K., Kasliwal, M. M., Hankins, M. J., et al. 2021, ApJ, 912, 19

de Jong, R. S., Bellido-Tirado, O., Chiappini, C., et al. 2012, in Society of PhotoOptical Instrumentation Engineers (SPIE) Conference Series, Vol. 8446, Ground-based and Airborne Instrumentation for Astronomy IV, ed. I. S McLean, S. K. Ramsay, \& H. Takami, 84460T

de Laverny, P., Recio-Blanco, A., Worley, C. C., \& Plez, B. 2012, A\&A, 544, A126

De Silva, G. M., Freeman, K. C., Bland-Hawthorn, J., et al. 2015, MNRAS, 449, 2604

Deepak, Lambert, D. L., \& Reddy, B. E. 2020, MNRAS, 494, 1348

Delgado Mena, E., Bertrán de Lis, S., Adibekyan, V. Z., et al. 2015, A\&A, 576, A69

Denissenkov, P. A., Ruiz, C., Upadhyayula, S., \& Herwig, F. 2021, MNRAS, 501, L33

Domogatskii, G. V., Eramzhian, R. A., \& Nadezhin, D. K. 1978, Ap\&SS, 58, 273

Duncan, D. K., Primas, F., Rebull, L. M., et al. 1997, ApJ, 488, 338

Ferrini, F., Matteucci, F., Pardi, C., \& Penco, U. 1992, ApJ, 387, 138

Feuillet, D. K., Feltzing, S., Sahlholdt, C. L., \& Casagrande, L. 2020, MNRAS, 497, 109

Fields, B. D., Olive, K. A., Yeh, T.-H., \& Young, C. 2020, J. Cosmology Astropart. Phys., 2020, 010

Franchini, M., Morossi, C., Di Marcantonio, P., et al. 2020, ApJ, 888, 55

Fu, X., Bressan, A., Molaro, P., \& Marigo, P. 2015, MNRAS, 452, 3256

Fu, X., Romano, D., Bragaglia, A., et al. 2018, A\&A, 610, A38

Fujimoto, S.-i., Matsuba, R., \& Arai, K. 2008, ApJ, 673, L51

Gaia Collaboration, Brown, A. G. A., Vallenari, A., et al. 2020, arXiv e-prints, arXiv:2012.01533

Gaia Collaboration, Prusti, T., de Bruijne, J. H. J., et al. 2016, A\&A, 595, A1

Gao, S., Liu, C., Zhang, X., et al. 2014, ApJ, 788, L37

Gao, S., Zhao, H., Yang, H., \& Gao, R. 2017, MNRAS, 469, L68

Gao, X., Lind, K., Amarsi, A. M., et al. 2020, MNRAS, 497, L30

Gilmore, G., Randich, S., Asplund, M., et al. 2012, The Messenger, 147, 25

Goudelis, A., Pospelov, M., \& Pradler, J. 2016, Phys. Rev. Lett., 116, 211303

Grisoni, V., Matteucci, F., Romano, D., \& Fu, X. 2019, MNRAS, 489, 3539

Grisoni, V., Romano, D., Spitoni, E., et al. 2020, MNRAS, 498, 1252

Grisoni, V., Spitoni, E., \& Matteucci, F. 2018, MNRAS, 481, 2570

Grisoni, V., Spitoni, E., Matteucci, F., et al. 2017, MNRAS, 472, 3637
Guiglion, G., Chiappini, C., Romano, D., et al. 2019, A\&A, 623, A99

Guiglion, G., de Laverny, P., Recio-Blanco, A., et al. 2016, A\&A, 595, A18

Heiter, U., Lind, K., Asplund, M., et al. 2015, Phys. Scr, 90, 054010

Helmi, A. 2020, ARA\&A, 58, 205

Hou, S. Q., He, J. J., Parikh, A., et al. 2017, ApJ, 834, 165

Iwamoto, K., Brachwitz, F., Nomoto, K., et al. 1999, ApJS, 125, 439

Izzo, L., Della Valle, M., Mason, E., et al. 2015, ApJ, 808, L14

Jackson, R. J., Jeffries, R. D., Wright, N. J., et al. 2020, MNRAS, 496, 4701

Jeffries, R. D., Jackson, R. J., Sun, Q., \& Deliyannis, C. P. 2021, MNRAS, 500, 1158

José, J. \& Hernanz, M. 2007, Journal of Physics G Nuclear Physics, 34, R431 Jurić, M., Ivezić, Ž., Brooks, A., et al. 2008, ApJ, 673, 864

Kelly, D. E., Christian, D. J., Mathioudakis, M., \& Jevremović, D. 2020, Research in Astronomy and Astrophysics, 20, 104

Khatri, R. \& Sunyaev, R. A. 2011, Astronomy Letters, 37, 367

Kirby, E. N., Guhathakurta, P., Zhang, A. J., et al. 2016, ApJ, 819, 135

Kusakabe, M., Cheoun, M.-K., Kim, K. S., et al. 2019, ApJ, 872, 164

Lambert, D. L. \& Reddy, B. E. 2004, MNRAS, 349, 757

Lanzafame, A. C., Frasca, A., Damiani, F., et al. 2015, A\&A, 576, A80

Lemoine, M., Vangioni-Flam, E., \& Cassé, M. 1998, ApJ, 499, 735

Limongi, M. \& Chieffi, A. 2018, ApJS, 237, 13

Lodders, K., Palme, H., \& Gail, H. P. 2009, Landolt Börnstein, 4B, 712

Luo, Y., Kajino, T., Kusakabe, M., \& Mathews, G. J. 2019, ApJ, 872, 172

Magrini, L., Lagarde, N., Charbonnel, C., et al. 2021, arXiv e-prints, arXiv:2105.04866

Matteucci, F., D’Antona, F., \& Timmes, F. X. 1995, A\&A, 303, 460

Matteucci, F., Molero, M., Aguado, D. S., \& Romano, D. 2021, arXiv e-prints, arXiv:2104.11504

Meneguzzi, M., Audouze, J., \& Reeves, H. 1971, A\&A, 15, 337

Michaud, G., Fontaine, G., \& Beaudet, G. 1984, ApJ, 282, 206

Molaro, P., Cescutti, G., \& Fu, X. 2020a, MNRAS, 496, 2902

Molaro, P., Izzo, L., Bonifacio, P., et al. 2020b, MNRAS, 492, 4975

Mucciarelli, A., Salaris, M., \& Bonifacio, P. 2012, MNRAS, 419, 2195

Nordlander, T., Korn, A. J., Richard, O., \& Lind, K. 2012, ApJ, 753, 48

Pancino, E., Lardo, C., Altavilla, G., et al. 2017, A\&A, 598, A5

Pasquini, L., Avila, G., Blecha, A., et al. 2002, The Messenger, 110, 1

Pitrou, C., Coc, A., Uzan, J.-P., \& Vangioni, E. 2018, Phys. Rep., 754, 1

Prantzos, N. 2012, A\&A, 542, A67

Prantzos, N., de Laverny, P., Guiglion, G., Recio-Blanco, A., \& Worley, C. C. 2017, A\&A, 606, A132

Ramírez, I., Meléndez, J., Bean, J., et al. 2014, A\&A, 572, A48

Randich, S., Gilmore, G., \& Gaia-ESO Consortium. 2013, The Messenger, 154, 47

Randich, S. \& Magrini, L. 2021, Frontiers in Astronomy and Space Sciences, 8, 6

Randich, S., Pasquini, L., Franciosini, E., et al. 2020, A\&A, 640, L1

Rebolo, R., Molaro, P., \& Beckman, J. E. 1988, A\&A, 192, 192

Recio-Blanco, A., de Laverny, P., Kordopatis, G., et al. 2014, A\&A, 567, A5

Reeves, H., Fowler, W. A., \& Hoyle, F. 1970, Nature, 226, 727

Romano, D., Chiappini, C., Matteucci, F., \& Tosi, M. 2005, A\&A, 430, 491

Romano, D., Franchini, M., Grisoni, V., et al. 2020, A\&A, 639, A37

Romano, D., Matteucci, F., Molaro, P., \& Bonifacio, P. 1999, A\&A, 352, 117

Romano, D., Matteucci, F., Ventura, P., \& D'Antona, F. 2001, A\&A, 374, 646

Romano, D., Matteucci, F., Zhang, Z.-Y., Ivison, R. J., \& Ventura, P. 2019, MN-

RAS, 490, 2838

Ruchti, G. R., Fulbright, J. P., Wyse, R. F. G., et al. 2011, ApJ, 743, 107

Sackmann, I. J. \& Boothroyd, A. I. 1999, ApJ, 510, 217

Sbordone, L., Bonifacio, P., Caffau, E., et al. 2010, A\&A, 522, A26

Schönrich, R., Binney, J., \& Dehnen, W. 2010, MNRAS, 403, 1829

Selvelli, P., Molaro, P., \& Izzo, L. 2018, MNRAS, 481, 2261

Shore, S. N. \& De Gennaro Aquino, I. 2020, A\&A, 639, L12

Sieverding, A., Langanke, K., Martínez-Pinedo, G., et al. 2019, ApJ, 876, 151

Sieverding, A., Martínez-Pinedo, G., Huther, L., Langanke, K., \& Heger, A.

2018, ApJ, 865, 143

Silva Aguirre, V., Ruchti, G. R., Hekker, S., et al. 2014, ApJ, 784, L16

Singh, R., Reddy, B. E., Bharat Kumar, Y., \& Antia, H. M. 2019, ApJ, 878, L21

Skrutskie, M. F., Cutri, R. M., Stiening, R., et al. 2006, AJ, 131, 1163

Smiljanic, R., Franciosini, E., Bragaglia, A., et al. 2018, A\&A, 617, A4

Smiljanic, R., Korn, A. J., Bergemann, M., et al. 2014, A\&A, 570, A122

Spada, F., Demarque, P., Kim, Y. C., Boyajian, T. S., \& Brewer, J. M. 2017, ApJ, 838,161

Spina, L., Randich, S., Magrini, L., et al. 2017, A\&A, 601, A70

Spite, F. \& Spite, M. 1982, A\&A, 115, 357

Starrfield, S., Bose, M., Iliadis, C., et al. 2020, ApJ, 895, 70

Starrfield, S., Truran, J. W., Sparks, W. M., \& Arnould, M. 1978, ApJ, 222, 600

Steigman, G. 2007, Annual Review of Nuclear and Particle Science, 57, 463

Tajitsu, A., Sadakane, K., Naito, H., Arai, A., \& Aoki, W. 2015, Nature, 518, 381

Taylor, C. J., Ritchey, A. M., Federman, S. R., \& Lambert, D. L. 2012, ApJ, 750, L15

Thompson, B. B., Few, C. G., Bergemann, M., et al. 2018, MNRAS, 473, 185 
Travaglio, C., Randich, S., Galli, D., et al. 2001, ApJ, 559, 909

Ventura, P., D’Antona, F., \& Mazzitelli, I. 2000, A\&A, 363, 605

Ventura, P., Dell'Agli, F., Lugaro, M., et al. 2020, A\&A, 641, A103

Ventura, P., Di Criscienzo, M., Carini, R., \& D’Antona, F. 2013, MNRAS, 431, 3642

Wallerstein, G. \& Sneden, C. 1982, ApJ, 255, 577

Wang, E. X., Nordlander, T., Asplund, M., et al. 2021, MNRAS, 500, 2159

Woosley, S. E., Hartmann, D. H., Hoffman, R. D., \& Haxton, W. C. 1990, ApJ, 356,272

Woosley, S. E. \& Weaver, T. A. 1995, ApJS, 101, 181

Yi, I. \& Narayan, R. 1997, ApJ, 486, 363

Yoshida, T., Suzuki, T., Chiba, S., et al. 2008, ApJ, 686, 448

Yuan, H., Liu, X., Xiang, M., Huang, Y., \& Chen, B. 2015, ApJ, 803, 13

1 INAF, Osservatorio di Astrofisica e Scienza dello Spazio, Via Gobetti 93/3, 40129 Bologna, Italy

e-mail: donatella.romano@inaf.it

2 INAF, Osservatorio Astrofisico di Arcetri, Largo E. Fermi 5, 50125 Firenze, Italy

3 Dipartimento di Fisica e Astronomia, Università degli Studi di Firenze, Via G. Sansone 1, 50019 Sesto Fiorentino, Firenze, Italy

${ }^{4}$ GEPI, Observatoire de Paris, Université PSL, CNRS, Place Jules Janssen, 92190 Meudon, France

5 Astrophysics Group, Keele University, Keele, Staffordshire ST5 5BG, UK

${ }^{6}$ Dipartimento di Fisica, Sezione di Astronomia, Università di Trieste, Via Tiepolo 11, 34131 Trieste, Italy

7 INAF, Osservatorio Astronomico di Trieste, Via Tiepolo 11, 34131 Trieste, Italy

${ }^{8}$ INFN, Sezione di Trieste, Via Valerio 2, 34127 Trieste, Italy

9 INAF, Osservatorio Astronomico di Padova, Vicolo dell'Osservatorio 5, 35122 Padova, Italy

${ }^{10}$ Leibniz-Institut für Astrophysik Potsdam (AIP), An der Sternwarte 16, 14482 Potsdam, Germany

11 Dipartimento di Fisica e Astronomia, Università degli Studi di Bologna, Via Gobetti 93/2, 40129 Bologna, Italy

12 INAF, Osservatorio Astronomico di Roma, Via Frascati 33, 00077 Monte Porzio Catone, Roma, Italy

13 SISSA, Via Bonomea 265, 34136 Trieste, Italy

14 Lund Observatory, Department of Astronomy and Theoretical Physics, Box 43, 22100 Lund, Sweden

15 Université Côte d'Azur, Observatoire de la Côte d'Azur, CNRS, Laboratoire Lagrange, Bd de l'Observatoire, CS 34229, 06304 Nice Cedex 4, France

16 Observational Astrophysics, Department of Physics and Astronomy, Uppsala University, Box 516, 75120 Uppsala, Sweden

17 School of Physics, UNSW, Sydney, NSW 2052, Australia

18 Centre of Excellence for All-Sky Astrophysics in Three Dimensions (ASTRO 3D), Australia

19 Institute of Theoretical Physics and Astronomy, Vilnius University, Sauletekio av. 3, 10257 Vilnius, Lithuania

20 Dipartimento di Fisica e Astronomia, Università di Padova, Vicolo dell'Osservatorio 3, 35122 Padova, Italy

21 Institute of Astronomy, University of Cambridge, Madingley Road, Cambridge CB3 0HA, UK

22 Núcleo de Astronomía, Facultad de Ingeniería, Universidad Diego Portales, Av. Ejército 441, Santiago, Chile

${ }^{23}$ Space Science Data Center-ASI, Via del Politecnico SNC, 00133 Roma, Italy

${ }^{24}$ Nicolaus Copernicus Astronomical Center, Polish Academy of Sciences, ul. Bartycka 18, 00-716 Warsaw, Poland

25 The Kavli Institute for Astronomy and Astrophysics at Peking University, 100871 Beijing, China

${ }^{26}$ Departamento de Física de la Tierra y Astrofísica and IPARCOSUCM, Instituto de Física de Partículas y del Cosmos de la UCM,
Facultad de Ciencias Físicas, Universidad Complutense de Madrid, 28040 Madrid, Spain

27 Departmento de Astrofísica, Centro de Astrobiología (INTA-CSIC), ESAC Campus, Camino Bajo del Castillo s/n, 28692 Villanueva de la Cañada, Madrid, Spain

28 INAF, Osservatorio Astronomico di Palermo, Piazza del Parlamento 1, 90134 Palermo, Italy

29 Max-Planck Institut für Astronomie, Königstuhl 17, 69117 Heidelberg, Germany 